chlorid $^{247}$ ); H. Ley und F. Werner ${ }^{248}$ ), über Schwermetallsalze sehr schwacher Säuren und Versuche zur Darstellung kolloidaler Metalloxyde; C. $\mathrm{L}$ arguierdes $\mathrm{B}$ a $\mathrm{n}$ c e $\mathbf{l} \mathbf{s}^{\mathbf{2 4 9}}$ ), über den Einfluß von Nichtelektrolyten auf Fällung von Kolloiden; L. V a n in o ${ }^{250}$ ), zur Geschichte des kolloidalen Goldes; R. Z s ig m on dy, über amikroskopische Goldkeime ${ }^{251}$ ) und über die Auslösung silberhaltiger Reduktionsgemische durch kolloidales Gold ${ }^{252}$ ), dann über Teilchengrößen in Hydrosolen $^{253}$ ); H. S i ed e n t o p f ${ }^{254}$ ), über kolloidale Alkalimetalle, A. M üll e r $^{255}$, über das Hydrosol des Thoriumoxydhydrats; $A$. Win kelblech ${ }^{256}$ ), zur Chemie der Kolloide; E. M üller und Fr. Spitzer ${ }^{257}$ ), über die Entwässerung von kolloidalem Kupferhydroxyd durch Elektroendosmose; E d. J o rd i s ${ }^{258}$ ), über kolloidale Kieselsäure; F. W. S ch m id t ${ }^{259}$ ), über das kolloidale, weiße Zinnoxysulfid.

Von Büchern und Monographien theoretisch-elektrochemischen und physikalischchemischen Inhalts, deren Erscheinen in das Berichtsjahr fällt, seien genannt: L e B lạnes wohlbekanntes Lehrbuch der Elektrochemie, in 4 . wesentlich vermehrter Auflage; $W$. Nernst, Theoretische Chemie, 5. Auflage, I. Hälfte; W. O s t w a ld, Lehrbuch der allgemeinen Chemie II, 3., (Verwandtschaftslehre II), erste Lieferung; F. W. $\mathrm{K}$ ü s t e r, Lehrbuch der allgemeinen, physikalischen und theoretischen Chemie, als Teil des im Neuerscheinen begriffenen $\mathrm{G} \mathrm{m}$ elin schen Handbuches; R. A b e g g, Handbuch der anorganischen Chemie (einzelne Lieferungen); v. J ü p t $\mathrm{n}$ e r, Lehrbuch der chemischen Technologie der Energien, das nun vollendet vorliegt; $W, \mathrm{H}$ e $\mathbf{r ~ z}^{260}$ ), Die Lehre von der chemischen Beschleunigung durch Fremdstoffe, (Katalyse); S. Arrhen i us, Theorien der Chemie; R. L o r e n z 261), Die Elektrolyse geschmolzener Salze, III. Teil: elektromotorische Kräfte; J. J. v a n L a a r, Sechs Vorträge über das thermodynamische Potential; O. S a c k u r, Úber die Bedeutung der Elektronentheorie für die Chemie; J. Mi e, Die neveren Forschungen über Ionen und Elektronen.

W i e n, im April 1907.

247) Siehe auch Compt. r. d. Acad. d. sciences 143, 1141 (1906).

248) Ber\}. Berichte 39, 2177 (1906). (1906).

249) Compt. r. d. Acad. id. sciences 143, 174

250) J. prakt. Chem. (2) 73, 575 (1906).

25I) Z. physikal. Chem. 56, 65 (1906).

252) Z. physikal. Chem. 56, 77 (1906).

253) Z. f. Elektrochem. 12, 631 (1906).

254) Z. f. Elektrochem. 12, 635 (1906).

255) Berl. Berichte 39, 2857 (1906).

256)) Diese Z. 19, 1953 (1906).

257) Z. f. Chem. u. Ind. d. Koll. 1, 44 (1906).

${ }^{258)} Z$ Z. f. Chem. u. Ind. d. Koll. 1, 97 (1906).

259) Z. f. Chem. u. Ind. d. Koll. 1, 129 (1906).

${ }^{260)}$ A h r e $n$ s sche Sammlung chem. u. chem.techn. Vorträge, Bd. 11.

261) E n gel h a r d t sche Monographien über angewandte Elektrochemie, Bd. 22.

\section{Über die Beziehungen \\ zwischen Lichtabsorption und chemischer Konstitution bei organischen Verbindungen.}

\author{
Von H. Lex-Leipzig. \\ (Eingeg. d. 5.(6. 1907.)
}

Auf den folgenden Seiten soll der Versuch gemacht werden, die bisher erkannten Beziehungen zwischen der Konstitution chemischer Verbindungen undihrerFarbedarzulegen. $\left.{ }^{1}\right)$ Bei der größerenMannigfaltigkeit, die die organischen Verbindungen aufweisen, ist es begreiflich, daß hior auf Grund eines größeren Beobachtungsmaterials und infolge der höher entwickelten Systematik die Beziehungen zwischen Konstitution und Farbigkeit relativ am besten erkannt sind; unsere Kenntnisse auf anorganischem Gebiete sowie auf dem der organischen Schwermetallverbindungen sollen später im $\mathrm{Zu}$ sammenhang vorgetragen werden.

\section{Allgemeines über Absorption.}

a) Absorption im sichtbaren Spektrum.

Die Farbe eines Stoffes kommt bekanntlich dadurch zustande, daß Lichtstrahlen bestimmter Wellenlänge von dem farbigen Stoff absorbiert werden. Zur genauen Untersuchung der genannten Eigenschaft betrachten wir den farbigen Stoff für sich oder in indifferenten Lösungsmitteln gelöst im durchfallenden Lichte. Unter diesen Umständen ist die Farbigkeit des Stoffes in erster Linie durch das ihm eigene Absorptionsspektrum bestimmt, das erhalten wird, indem man homogenes weißes Licht durch den festen oder gelösten Stoff hindurchfallen läßt und das hindurchgegangene Licht spektralanalytisch untersucht. Je nach der Art der Absorption kann man etwa 4 Gruppen von Absorptionsspektren unterscheiden :

1. Einseitige Absorptionsspektra, bei denen nur die eine Hälfte, meist die blaue absorbiert wird (Pikrinsäure, Eisenchlorid).

2. Zweiseitige Absorptionsspektra, bei denen nur Strahlen von mittlerer Wellenlänge, grüne oder grünblaue durchgelassen werden (Lösungen von Kupferchlorid [konz.] oder Nickelchlorür).

3. Bandenspektra, bei denen innerhalb des sichtbaren Spektrums mehr oder weniger dunkle Banden auftreten (Kobaltchlorür). ?

4. Linienspektra, bei denen einzelne dunkle Absorptionslinien sichtbar sind (Joddampf, Lösungen von Didymsalzen).

In fast allen Fällen ist das Absorptionsspektrum nicht allein von der stofflichen Natur der gelösten Verbindung, sondern auch von äußeren Bedingungen abhängig; als solche sollen die Dicke der durchstrahlten Schicht, die Konzentration des gelösten Stoffes, die Natur des Lösungsmittels und die Temperatur genannt werden. (Näheres hierüber siehe auch Kap. 5.)

1) Wobei in erster Linie die neueren Arbeiten, für die auch die Literatur angegeben wurde, berücksichtigt werden sollen; in manchen Fällen, so besonders in der Einleitung, mußte auch auf die ältere Literatur zurückgegriffen werden. 
Wie später ausführlich gezeigt werden soll, besitzen viele, besonders aromatische Verbindungen, Absorptionsbanden im ultravioletten Teile des Spektrums; durch Einführung bestimmter Atom. gruppen erfolgt häufig eine Verschiebung dieser Banden in das Gebiet des sichtbaren Spektrums, also zunächst ins Violett, wodurch der Stoff in der Komplementärfarbe d. h. grüngelb erscheint. Bekanntlich wird die physiologische Empfindung des Weiß durch Mischung je zweier komplementärer Farben des Spektrums, z. B. von Violett und Grüngelb, Indigo und Gelb usw., hervorgebracht, wie das in dieser Skizze (Fig. 1) veranschaulicht werden soll.

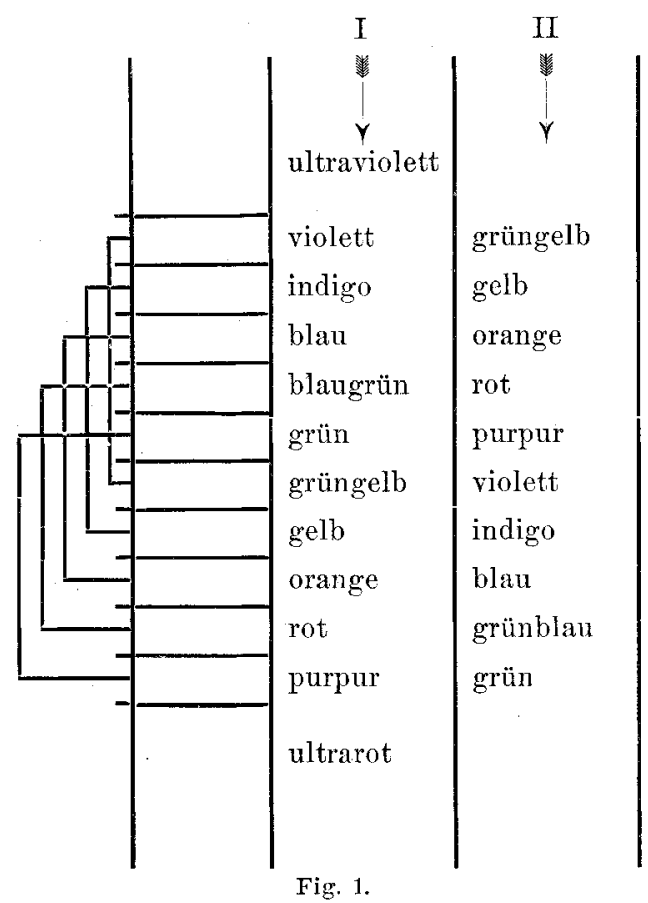

Ist man nun imstande durch sukzessive Einführung gewisser Gruppen in das Molekül des ursprünglich ,farblosen Stoffes" das Absorptionsband aus dem ultravioletten gegen das rote Ende des Spektrums zu verschieben $(-I)$, so ändert sich die Farbe des Stoffes allmählich von grüngelb über blau bis grün, wie die beifolgende Skizze ergibt (- II). Grüngelb erscheint somit als primitivste Farbe. Wir wollen mit S c h ü t z e den Übergang von Gelb nach Grün als $\mathbf{F}$ a $\mathrm{rbverti}$ ef $\mathbf{u} \mathbf{n} \mathrm{g}$, den entgegengesetzten als $\mathrm{F}$ arberhöhung bezeichnen. Gruppen, welche farbvertiefend wirken, sollen bathoc h r o me, solche, die Farberhöhung verursachen, hy p s o hrome genannt werden. Schließlich sei noch erwähnt, daß die geschilderte Reihenfolge in der Farbänderung dadurch häufig gestört wird, da $\beta$ mehrere Absorptionsbanden aus dem Ultraviolett heraustreten und zwar die zweite Bande früher erscheint, ehe die erste über das sichtbare Rot hinweggeschritten ist.

b) Absorption im unsichtbaren Teil des Spektrums.

Früher beschränkten sich die Untersuchungen des Absorptionsspektrums lediglich auf den dem Auge sichtbaren Teil des Spektrums, d. h. auf Wellenlängen, die etwa zwischen 820 und $375 \mu \mu$ liegen.
Neuere Untersuchungen, die man besonders $\mathrm{H}$ a $\mathbf{r} \mathrm{t}$. le y (1879) verdankt, haben den Beweis geliefert, daß viele Stoffe im äußersten Ultraviolett ${ }^{2}$ ) selektive Absorption zeigen und somit im weiteren Sinne auch als ,farbig" zu bezeichnen sind und von Wesen, die mit anderen Augen ausgestattet sind, auch als farbig empfunden werden müßten.

Hinsichtlich der Absorption im. Ultraviolett teilt $\mathrm{H}$ artle y die Stoffe in drei Klassen ein :

1. Stoffe, die am ultravioletten Ende absorbieren, aber durch Verdünnung mit indifferenten Lösungsmitteln leicht durchlässiger gemacht werden können. (Verbindungen mit offener Kohlenstoffkette, Paraffine, Olefine, Acetylenkohlenwaserstoffe ; Substitution eines oder mehrerer Wasserstoffatome durch $(\mathrm{OH}),\left(\mathrm{CO}_{2} \mathrm{H}\right),\left(\mathrm{OCH}_{3}\right),\left(\mathrm{NH}_{2}\right)$ ändert nicht den Charakter des Spektrums, sondern nur das Absorptionsvermögen.)

2. Stoffe, die ähnlich wie unter 1. angegeben, aber stärker absorbieren, sodaß Verdünnung geringeren Einfluß hat; hierhin gehören Verbindungen mit geschlossener Kohlenstoffkette (Furfuran, Thiophen, Pyrrol, Piperidin, Campher, hydrierte Benzole usw.).

3. Stoffe, die bei großem Absorptionsvermögen deutliche Absorptionsstreifen hervorrufen (Benzol, Naphthalin, Pyridin, Chinolin, Pyrazin usw.).

Wasser und Methylalkohol sind äußerst durchlässig für kurzwellige Strahlen, so daß diese als Lösungsmittel für andere im Ultraviolett absorbierenden Stoffe benutzt werden können. Messungen im Ultraviolett sind für die theoretische Behandlang des Absorptionsproblems von fundamentaler Bedeutung, worauf noch kürzlich von $A$. v. $B$ a e y e $r^{3}$ ) nachdrücklich hingewiesen wurde.

Sehen wir z. B. einen für unser Auge farblosen Stoff durch eine chemische Veränderung (Einführung neuer Gruppen, Salzbildung usw.) gelb werden, so kann die Farbänderung entweder kontinuierlich sein : indem eine hart am sichtbaren Teil des Spektrums liegende Absorptionsbande aus dem Ultravioletten ins Violette rückt; oder aber diskontinuierlich : indem sich eine im äußersten Ultraviolett befindliche Bande in das sichtbare Gebiet verschiebt. Es erscheint die Annahme plausibel, daf im ersten Falle eine geringe, im zweiten Falle dagegen eine durchgreifende Änderung in der Konstitution des Moleküls vor sich geht.

Andererseits kennen wir auch Stoffe, die im Gebiete der langen Wellenlängen, $\mathrm{d}$. h. im Ultrarot selektive Absorption aufweisen. Hier sind jedoch die Untersuchungen noch nicht so weit fortgeschritten, daß durchsichtige Beziehungen zwischen Absorption und Konstitution erkennbar wären. (Vgl. darüber $\mathrm{K}$ a y s e r, Handbuch der Spec. troscopie III, 304.)

\section{Chromophortheorie.}

Schon früher hatte man erkannt, daß zwischen Absorptionsspektrum und chemischer Konstitution gewisse Beziehungen bestehen. Die ersten Versuche nach dieser Richtung rühren von $G \mathbf{r}$ a e b e und $\mathrm{L}$ i e b e rman her und erreichten in der von

2) Näheres siehe Ka yser, Handbuch der Spectroscopie, Bd. 3.

3) Diese Z. 19, 1287 (1906). 
W i t $t$ aufgestellton Chromophortheorie einen vorläufigen Abschluß. Diese Theorie macht bekanntlich für die Absorption eines farbigen Stoffes gewisse Atomgruppen verantwortlich, die $\mathrm{Ch}$ r o m o ph o r e genannt werden und deren Einführung in farblose Moleküle selektive Absorption im sicht. baren Spektrum erzeugt. Wenn von weniger wichtigen abgesehen wird, so sind folgende Atomgruppierungen als Chromophore erkannt $\left.{ }^{4}\right)$ :

1. Aethylengruppen $>\mathrm{C}_{-}^{-} \mathrm{C}<$ (in bestimmter Zahl und Lagerung).

2. Carbonylgruppen - $\mathrm{CO}$

3. Stickstoffkohlenstoffgruppierungen $>\mathrm{C}_{--}^{-} \mathrm{NH}$ resp. $-\mathrm{CH}_{-}^{-} \mathrm{N}-$

4. Azogruppen - $\mathrm{N}_{-}^{-} \mathrm{N}$

5. An Kohlenstoff gebundene Nitrosogruppen $\mathrm{YC}-\mathrm{NO}$

6. Nitrogruppen -- $\mathrm{NO}_{2}$.

SchlieBlich zeigen noch verschiedene schwefelhaltige Gruppen, wie $-\mathrm{S}_{2}-$, chromophorische Wirkungen, auch die COOH- resp. COOR-Gruppe scheint im Vereine mit anderen Gruppen z. B. Phenyl für die Ausbildung eines Absorptionsstreifens im Violett günstig zu wirken.

Es ist ohne weiteres verständlich, daß die mehrmalige Einführung chromophorer Gruppen im allgemeinen den Effekt verstärken, d. h. die Farbe vertiefen und daB auch die gegenseitige Lage der Chromophore einen Einfluß ausüben wird. Ferner werden wir a priori annehmen dürfen, da 3 die Nähe der Chromophore bzw. ihre dichte Gruppierung die Farbigkeit günstig beeinflussen wird; schließlich wird sich aus dem folgenden ergeben, daß eine Kombination verschiedener Chromophore in demselben Moleküle zu neuen z. B. chinoiden Gruppen häufig farberregend wirkt.

\section{Die Aethylengruppe: $>\mathrm{C}_{-} \mathrm{C}<$}

Beispiele einfacher farbiger Athylenderivate etwa von dem Typus $\mathrm{R}_{2} \mathrm{C}: \mathrm{CR}_{2}$ sind nicht bekannt. Farbe tritt erst auf, wenn mehrere Athylenbindungen in eyclischer Anordnung vorhanden sind. Das prägnanteste Beispiel ist das von $\mathrm{Th}$ i e le entdeckte Fulven (I) sowie die substituierten Fulvene z. B. Diphenylfulven (II)<smiles>C=C1C=CC=C1</smiles>

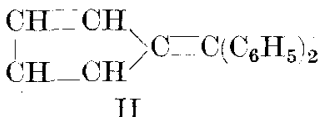

Das orangegelbe Fulven ist isomer mit dem farblosen Benzol (III)

$$
\begin{gathered}
\mathrm{CH} \text { CH-CH } \\
\mathrm{CH} \text { CH-CH } \\
\text { III }
\end{gathered}
$$

eine Tatsache, die plausibel erscheint, da, die Gültigkeit der $\mathrm{K}$ e $\mathrm{k} \mathrm{u} l$ é schen Formel des Benzols vorausgesetzt, in diesem die Athylenbindungen weniger dicht gelagert sind als im Fulven, wie die Betrach-

4) Im wesentlichen folgen wir hier der ausführlichen und verdienstvollen Zusammenstellung von H. K a u f $\mathrm{fm}$ a $\mathrm{n} \mathbf{n}$,Über den Zusammenhang zwischen Farbe und Konstitution" Ahrens, Sammlung, Bd. IX. tung der Schemata I und III sofort ergibt; auch könnte die weniger symmetrische Anordnung der Doppelbindungen im Fulven in gewissem Grade mitbestimmend für dessen Farbigkeit sein. Dennoch ist Benzol in w e iterem Sinne $\mathrm{farbi}_{\mathrm{g}} \mathrm{zu}$ nennen, da dieser Stoff im Ultraviolett selektive Absorption zeigt, für die 7 Absorptionsbanden charakteristisch sind. Dasselbe gilt von den Verbindungen mit kondensierten Benzolringen wie Naphthalin mit 4 Absorptionsbanden; ferner von denen mit heterocyclischen Ringsystemen wie Pyridin, Chinolin und Pyrazin.

Die Gegenwart der drei Athylenbindungen im Benzol bedingt es, daß die Einführung dieses Ringsystems in farblose Verbindungen häufig das Auftreten von Absorptionsbanden im Violett hervorruft, wie die Beobachtungen von $S t o b b e^{5}$ ) an den Fulgensäuren sowie deren Anhydriden beweisen. Die noch nicht isolierte Stammsubstanz (I), Butadiendicarbonsäure, ist nach Analogien farblos, da auch

$$
\begin{array}{cc}
\mathrm{H}_{2} \mathrm{C}^{-} \mathrm{C}-\mathrm{COOH} & \left(\mathrm{CH}_{3}\right)_{2} \mathrm{C}_{-} \mathrm{C}-\mathrm{COOH} \\
\mathrm{H}_{2} \mathrm{O}-\mathrm{C}-\mathrm{COOH} & \left(\mathrm{CH}_{3}\right)_{2} \mathrm{C}_{-} \mathrm{C}-\mathrm{COOH} \\
\mathrm{I} & \mathrm{II} \\
\left(\mathrm{C}_{6} \mathrm{H}_{5}\right)_{2} \mathrm{C}_{-} \mathrm{C}-\mathrm{COOH} & \left(\mathrm{C}_{6} \mathrm{H}_{5}\right)_{2} \mathrm{O}_{-} \mathrm{C}-\mathrm{COOH} \\
\left(\mathrm{C}_{6} \mathrm{H}_{6}\right) \mathrm{HC}^{\mathrm{C}}-\mathrm{COOH} & \left(\mathrm{C}_{6} \mathrm{H}_{5}\right)_{2} \mathrm{C}-\mathrm{C}-\mathrm{COOH} \\
\text { III } & \text { IV }
\end{array}
$$

die tetramethylierto Säure farblos ist, während Triphenylfulgensäure (III) gelb und Tetraphenylfulgensäure (IV) orange ist. Eine noch stärkere Farbvertiefung erleiden die Anhydride der Fulgensäuren, die Fulgide, durch sukzessive Einführung von Phenylresten.

\section{Die Carbonylgruppe - CO}

besitzt deutlich chromophoren Charakter; zwar sind die cinfachen Aldehyde und Ketone $\mathrm{R}-\mathrm{CO}-\mathrm{H}$ und $\mathbf{R}-\mathbf{C O}-\mathbf{R}$ farblos, jedoch schon die aliphatischen $a$-Diketone und mehr noch die Triketone lebhaft farbig, z. B.

$\mathrm{CH}_{3} \mathrm{CO} . \mathrm{CO} . \mathrm{CH}_{3}$ : gelb, $\mathrm{CH}_{3} \mathrm{CO} . \mathrm{CO} . \mathrm{CO} . \mathrm{CH}_{3}$; orange $\mathrm{C}_{6} \mathrm{H}_{5} \mathrm{CO} . \mathrm{CO} . \mathrm{CO} . \mathrm{C}_{6} \mathrm{H}_{5}$ : gelb. Die nochmalige Einführung einer Carbonylgruppe hat eine weitere $\mathrm{Farb}$ vertiefung im Gefolge; $\mathrm{C}_{6} \mathrm{H}_{5} \mathrm{CO} . \mathrm{CO} . \mathrm{CO} . \mathrm{COO}_{6} \mathrm{H}_{5}$ ist wasserfrei rot und bildet ein gelbes Hydrat.

Als weitere Beispiele seien genannt:

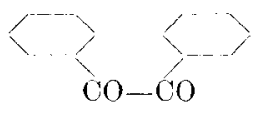

Benzil, gelb,

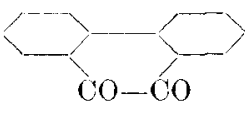

Phenanthrenchinon, orange.
Sie beweisen, daß die gleichzeitige Ausbildung eines isocyclischen Ringes (z. B. des mittleren Sechsringes im Phenanthrenchinon) die chromophoren Wirkungen der -CO--CO-Gruppe wesentlich verstärkt, worauf von $K$ a $\mathbf{u} f \mathrm{f} \mathbf{m} \mathbf{a} \mathbf{n}$ und neuerdings von $\mathrm{S}$ t o b b e aufmerksam gemacht wurde. Als weitere Belege für diese Tatssache seien

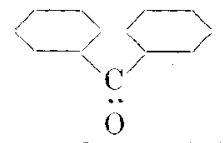

Benzophenon, farblos, und

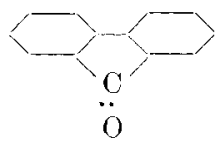

Fluorenon, orange,
5) Besonders Liebigs Ann. 349, 333. 
sowie<smiles>C1CCC2CCCC[C-](C3C4CCCCC(CCCC5CCCCCC53)C4)C2CC1</smiles>

Tetraphenyläthylen und

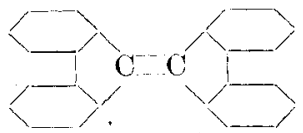

Bibisphenylenäthen, rot,

genannt; im letzteren Falle sehen wir durch die zweimalige Ringschließung die chromophoren Wirkungen der mittleren Athylengruppe in ganz auffälliger Weise erhöht.

Der Carbonylgruppe verwandt ist die

3. $\mathrm{G}$ r u p p e $\mathrm{C}_{-}^{-} \mathrm{N}$-, deren Vorhandensein in Benzylidenanilin : $\mathrm{C}_{6} \mathrm{H}_{5} \mathrm{CH}: \mathrm{NC}_{6} \mathrm{H}_{5}$, Benzophenonphenylimin : $\left(\mathrm{C}_{6} \mathrm{H}_{5}\right)_{2} \mathrm{C}: \mathrm{NC}_{6} \mathrm{H}_{5}$ Diacetyldianil : $\mathrm{CH}_{3} \mathrm{C}\left(: \mathrm{NC}_{6} \mathrm{H}_{5}\right) \mathrm{C}\left(: \mathrm{NC}_{6} \mathrm{H}_{5}\right) \mathrm{CH}_{3}$ u. a. die gelbe Farbe dieser Verbindungen bedingt.

Wird in der Gruppe - CR - NR (-CR) "' durch ein äquivalentes Stickstoffatom $(\mathrm{N})^{\prime \prime \prime}$ ersetzt, so entsteht

4. die A z ogruppe-N-N-- mit stark chromophorem Charakter, der somit in der Reihe:

$$
\cdot \mathrm{CR}_{\sim-}^{-} \mathrm{CR} \cdot, \cdot \mathrm{CR}_{\sim}-\mathrm{NR}, \cdot \mathrm{N}_{-}^{--} \mathrm{N} \text {. }
$$

also mit steigendem Stickstoffgehalt meist sehr erheblich zunimmt, denn schon fette Azoverbindungen wie

$$
\mathrm{CH}_{2} \backslash \stackrel{N}{N}
$$

Diazomethan

$$
\text { und }
$$$$
\begin{aligned}
& \mathrm{N} \cdot \mathrm{COOCC}_{2} \mathrm{H}_{5} \\
& \mathrm{~N} \cdot \mathrm{COOC}_{2} \mathrm{H}_{5}
\end{aligned}
$$

Azodicarbonsäureester

sind stark gelb. Azobenzol $\mathrm{C}_{6} \mathrm{H}_{5}-\mathrm{N}_{-}-\mathrm{NC}_{6} \mathrm{H}_{5}$ und Homologe, sowie deren einfache Substitutionsprodukte, die Grundsubstanzen des ungeheuren Heeres der Azofarbstoffe sind orange bis rot. Merkwürdigerweise sind manche echte aliphatische Azoverbindungen der Isobuttersäurereihe farblos. Nach $\mathrm{T} h$ i e l e und $\mathrm{He} \mathrm{u}$ s e ${ }^{6}$ ), den Entdeckern dieser Verbindungen, treten die chromophoren Wirkungen der Azogruppe dann besonders in die Erscheinung, wenn mit derselben negative Gruppen in direkter Bindung stehen:

$$
\begin{aligned}
& \mathrm{N} \cdot \mathrm{COOH} \mathrm{N} \cdot \mathrm{CO} \cdot \mathrm{NH}_{2} \\
& \stackrel{\mathrm{N} \cdot \mathrm{COOH}}{ }, \| \mathrm{N} \cdot \mathrm{CO} \cdot \mathrm{NH}_{2}: \text { gelb } \\
& \mathrm{N} \cdot \mathrm{C}\left(\mathrm{CH}_{3}\right)_{2} \mathrm{CN} \quad \mathrm{N} \cdot \mathrm{C}\left(\mathrm{CH}_{3}\right)_{2} \cdot \mathrm{COOH} \\
& \mathrm{N} \cdot \mathrm{C}\left(\mathrm{CH}_{3}\right)_{2} \mathrm{CN}^{\prime} \stackrel{\|}{\mathrm{N}} \cdot \mathrm{C}\left(\mathrm{CH}_{3}\right)_{2} \cdot \mathrm{COOH} \text { : farblos. }
\end{aligned}
$$

Es hätte ein großes Interesse, diese Verbindungen auf Absorption im äußersten Violett und im Ultraviolett zu untersuchen. Wesentlich schwächer scheint der chromophore Charakter der

5. Azox ygrup pe

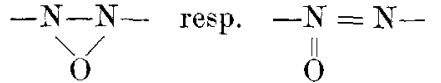

zu sein, denn die Verbindungen mit diesen Chromophor besitzen durchwegs. hellere Farbe als die Azokörper.

6. Die $\mathrm{Nitrosogruppe-N-O} \mathrm{übt} \mathrm{äu-}$ Berst starke chromophore Wirkungen aus, falls sie direkt an Kohlenstoff gebunden ist. Schon die aliphatischen echten Nitrosoverbindungen wie Nitrosotertiärbutan $\mathrm{ON}, \mathrm{C}\left(\mathrm{CH}_{3}\right)_{3}$ sind in Lösung oder in geschmolzenem Zustande, wo sie in monomoleku. larer Form vorhanden sind tiefblau; Nitrosobenzol

6) Liebigs Ann. 290, 6.

$\mathrm{ON} . \mathrm{C}_{6} \mathrm{H}_{5}$ ist unter gleichen Bedingungen grün, hier vereinigen sich die Wirkungen der Nitrosogruppe und der drei Athylenbindungen des Benzols, um die weitere Farbvertiefung nach Grün zu er. zeugen.

Die an Stickstoff gebundene Nitrosogruppe z. B in $\mathrm{C}_{6} \mathrm{H}_{5} \mathrm{~N}\left(\mathrm{CH}_{3}\right)$. NO hat nur sehr geringe farbgebende Eigenschaften. Gering ist ferner der Effekt der

7. Nitrogruppe $\mathrm{NO}_{2}$, der die Konstitution<smiles>C[As](C)(=O)O</smiles>

zukommt. (s. später). Nitronaphthalin ist schwach gelblich und kann als Schulbeispiel für das Auftreten von Farbe gelten: Naphthalin besitzt, wie früher erwähnt, Absorptionsbanden im Ultraviolett, durch Einführung der Nitrogruppe rücken diese ins Violett, die Substanz erscheint gelblich. Der chromophore Charakter der Nitrogruppe ist früher stark überschätzt worden, so ist die gelbe Farbe des Nitrophenols nicht allein auf die Anwesenheit der Nitrogruppe zurückzuführen, sondern auch durch intramolekulare Umlagerung zu erklären, wie später begründet werden wird.

8. Der $\mathrm{Ch}$ romophor $-\mathrm{N} O$ mit vier-

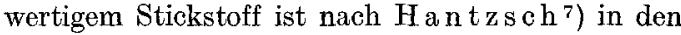
stickoxydsulfonsauren Salzen z. B. $\mathrm{O}: \mathrm{N}:\left(\mathrm{SO}_{3} \mathrm{~K}\right)_{2}$ enthalten, die in festem Zuistande intensiv gelb, in gelöstem Zustande violett sind; wahrscheinlich verdankt auch das braune Stickstoffdioxyd $\mathrm{O}: \mathrm{N}: \mathrm{O}$ seine intensive Farbe der Anwesenheit dieses Chromophors, der nach $\mathbf{P}$ il ot $\mathbf{y}^{8}$ ) auch die rote Farbe der Porphyrexide z. B.

$$
\begin{aligned}
& \left(\mathrm{CH}_{3}\right)_{2} \mathrm{C}-\mathrm{N}=\mathrm{O} \\
& \mathrm{HN}=\mathrm{C}-\mathrm{NH}=\mathrm{NH}
\end{aligned}
$$

bedingen soll.

9. Die übrigens weniger wichtige $\mathrm{Th}$ i o. c a r b on y lg r u p pe : $\mathrm{C}_{-}^{-} \mathrm{S}$ hat wesentlich stär. kere chromophore Eigenschaften, als ihr sauerstoff haltiges Analogon: Thioacetphenon $\mathrm{C}_{6} \mathrm{H}_{5} \mathrm{CSCH}_{3}$ und Thiobenzophenon $\mathrm{C}_{6} \mathrm{H}_{5} \mathrm{CSC}_{6} \mathrm{H}_{5}$ stellen blaue Ole $\operatorname{dar}^{9}$ ).

10. Chinoide Chromophore. Eine beträchtliche Verstärkung erfahren die farbgebenden Eigenschaften der Carbonylgruppe durch gleichzeitige Anwesenheit von Doppelbindungen. Einen Beleg hierfür bilden die neuerdings entdeckten Ketene ${ }^{10}$ ) z. B. $\left(\mathrm{CH}_{3}\right)_{2} \mathrm{C}_{-}^{--} \mathrm{C}_{--}^{--} \mathrm{O}$ und $\left(\mathrm{C}_{6} \mathrm{H}_{5}\right)_{2} \mathrm{C}_{-}^{--} \mathrm{C}-\mathrm{O}$, die gelbe Öle darstellen, ferner das gelbe Phoron: $\left(\mathrm{CH}_{3}\right)_{2} \mathrm{C}=\mathrm{CH} . \mathrm{CO} . \mathrm{CH}_{-}^{-} \mathrm{C}\left(\mathrm{CH}_{3}\right)_{2}$, in dem die Atomgruppierung :

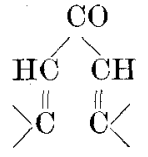

die Farbigkeit hervorruft. Denken wir uns die endstelligen Kohlenstoffatome durch eine weitere $\mathrm{CO}$.

7) Berl. Berichte 28, 2744.

8) Berl. Berichte 36, 1283.

9) Berl. Berichte 28, 895; 29, 2974.

$\left.{ }^{10}\right)$ S t a u ding e r, Berl, Berichte 38, 1735 39, 968 , 
Gruppe zum Ringe geschlossen, so gelangen wir zum p-Chinon $(\mathrm{I})$, dessen Farbigkeit durch die gleich-<smiles>CC1C=CC(C=O)C=C1</smiles><smiles>O=C1C=CC=CC1=O</smiles>

zeitige Wirkung zweier $\mathrm{C}_{--}^{-} \mathrm{O}$ - und zweier $\mathrm{HC}_{-}^{-\cdots} \mathrm{CH}-$ Gruppen plausibel erseheint. Auf Grund der früheren Uberlegungen ist auch die tiefere Farbe des durch Willstätters ${ }^{11}$ ) Untersuchungen genauer bekannt gewordenen o-Chinons (II) im Gegensatz zum p-Chinon nicht weiter rätselhaft, denn in ersterem sind beide Chromophore, sowohl die beiden $\mathrm{CO}$ als auch die $\mathrm{CH}-\mathrm{CH}$-Gruppen in größerer Nähe. Die chinoide Atomgruppierung, die sich durch die Formeln<smiles>C=CC=CC(=C)C=CC=C</smiles>

p-Chinoid und

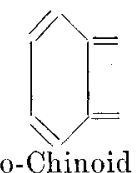

veranschaulichen Iäßt, wird bekanntlich im Sinne der Chromophortheorie als letzter Grund für die Farbigkeit selbst komplizierter Farbstoffe angesehen; hier muß auf die einschlägigen Arbeiten $\mathrm{N}$ ietzkis, Bernthsens, Kehrmanns u. a. hingewiesen werden. Auch die Existenz von $m$-Chinoiden ist neuerdings sehr wahrscheinlich gemacht worden (s. S. 12). Eine weitere Häutung der Chromophore sehen wir in W il ls t $\ddot{a}$ t t e r s ${ }^{12}$ ) Diphenochinon

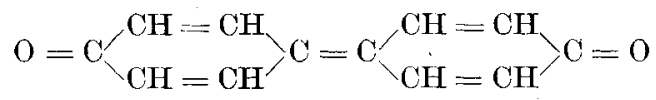

das in zwei Formen, einer gelben und roten existiert. Wird im p-Chinon die CO-Gruppe durch $\mathrm{CH}_{2}-\mathrm{C}<$ ersetzt, so bleibt, weil der ungesättigte Charakter des Moleküls (s. spätcr) nicht verringert wird, die Farbigkeit bestehen, was durch das Diphenylchinomethan (Fuchson)

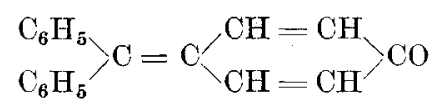

$\mathrm{B}$ i s t r z y c k i s 13) illustriert wird, das goldgelbe Farbe aufweist. Auch die Fulvene enthalten die für die Chinone charakteristische Atomgruppierung:

$$
\begin{array}{r}
\mathrm{C}=\mathrm{C}^{\prime} \\
\mathrm{C}=\stackrel{\mathrm{C}}{\mathrm{C}}
\end{array},
$$

sie sind nach $\mathrm{Thiele}$ gewissermaßen "halbe Chinone".

Es erscheint immerhin auffällig, daß die von $\mathrm{Z}$ in c ke, A u wers und B a $\mathrm{m}$ ber ger studierten Chinole ${ }^{14}$ ) z. B.

11) Berl. Berichte 3\%, 4744

12) Berl. Berichte 38, 1232.

13) Berl. Berichte 36, 2335.

14) Siehe besonders Berl. Berichte 33, $3600 \mathrm{ff}$. ferner Berl. Berichte 40, 1893, woselbst weitere Lit.
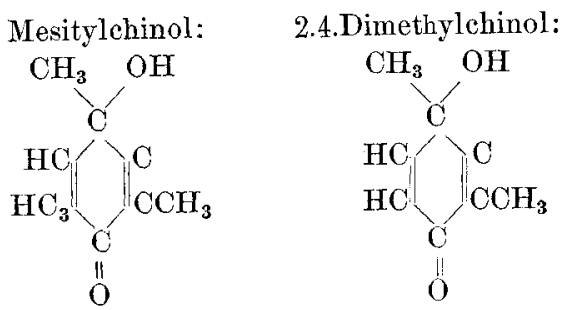

völlig farblose Verbindungen darstellen, obwohl sie ebenfalls drei Doppelbindungen in derselben Anordnung wie im Fulven enthalten und halbe Chinone genannt werden könnten.

Die Farbigkeit der Fulgide, der Anhydride der Fulgensäuren<smiles>[R12]C=C1C(=O)OC1=O</smiles><smiles>C1CCOC1</smiles>

Tetrahydrofuran

führt $S$ t o b b e ${ }^{15}$ ) darauf zurück, daß diese Verbindungsklasse als Doppelchinoide und zwar als pund o-Chinone des 'Tetrahydrofurans anzusehen sind, und erblickt als Träger der Fulgidfarbe die nur benachbarten ringförmig angeordneten ungesättigten Radikale,

Nach dem früheren ${ }^{16}$ ) sollten wir erwarten, daß Ersatz des Sauerstoffs durch die äquivalente Gruppe (NR)" die Farbigkeit des Chinons erhöhen würde; in der Tat ist Chimondiphenylimid :

$$
\mathrm{C}_{6} \mathrm{H}_{5} \mathrm{~N}=\Longrightarrow=\mathrm{NC}_{6} \mathrm{H}_{5}
$$

braunrot, hingegen sind die einfachsten Chinonimide wie $\mathrm{NH}: \mathrm{C}_{6} \mathrm{H}_{4}: \mathrm{NH}$ und $\mathrm{O}: \mathrm{C}_{6} \mathrm{H}_{4}: \mathrm{NH}$ farblos, Chinondimethylimin : $\mathrm{CH}_{3} \mathrm{~N}: \mathrm{C}_{6} \mathrm{H}_{4}: \mathrm{NCH}_{3}$ ist im festen Zustande farblos und nur in Lösung hellgelb; auch die Salze der einfachen Chinonimide wie $\mathrm{NH}: \mathrm{C}_{6} \mathrm{H}_{4}: \mathrm{NH}_{2} \mathrm{Cl}$ sind nach $\mathrm{Wi} 11$. s t ät t e r ${ }^{17}$ ), dem man diese Untersuchungen verdankt, farblos. Diese Tatsachen müssen überraschen, da manche Chinonimoniumsalze wie $\mathrm{HN}: \mathrm{C}_{6} \mathrm{H}_{4}: \mathrm{N}\left(\mathrm{CH}_{3}\right)_{2} \mathrm{Cl}$ farbig sind. $\mathrm{Ob}$ es sich hier um feinere Isomerien innerhalb des Moleküls des Chinondiimids handelt, derart, dah dieses in einer farbigen und farblosen Form auftreten kann, müssen weitere Untersuchungen lehren.

Sehr häufig finden wir chinoide Atomgruppierungen innerhalb eines Ringsystems. Als o-Chinoide seien genannt:<smiles>CCC1=NC(CC)C(CC)=C(CC)C2CCCCC12</smiles>

Von p Chinoiden erwähnen wir:

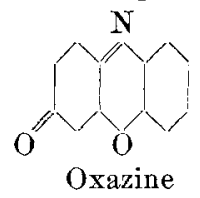

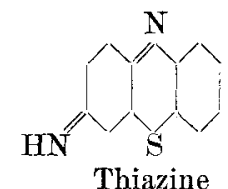

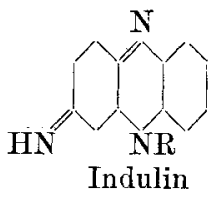

15) Liebigs Ann. 349, 361 .

16) s. S. 1306.

17) Berl. Berichte 3\%, 1494, 3761, 4605; 38, 2244. 

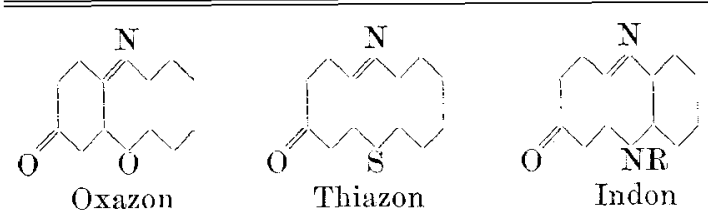

Wie die umfassenden Cntersuchungen von $O$. $\mathrm{F}$ is $\mathrm{eh}$ er und $\mathrm{Hepp}, \mathrm{Bernth}$ sen, $\mathrm{K}$ ehr. m a n n, Niet zki, Witt u. a. gelehrt haben, leiten sich von diesen Ringsystemen eine große Zahl der wichtigsten Farbstoffe ab, auf die einzugehen außerhalb des Rahmens dieser Betrachtungen liegt.

Wie früher beiläufig erwähnt, wird die chromophore Wirkung gewisser Gruppen häufig durch gleichzeitige Ringschließung unterstützt. Wie eine Untersuchung S tob bes ${ }^{18}$ ) an der Hand eines großen Materials aber erwiesen hat, tritt bei kohlenstoff-, wasserstoff- und sauerstoffhaltigen Verbin. dungen Farbvertiefung nur dann auf, wenn eine isocyclische Gruppierung entsteht; z. B.

$$
\mathrm{CH}_{\mathrm{CH}}^{\mathrm{CH}}=\mathrm{CHC}_{6} \mathrm{H}_{5}
$$

$\alpha$ - $\delta$-Diphenylbutadien, farblos,<smiles>CCCCC1CCCCC1CCCC</smiles>
$\mathrm{CH}$ Benzalinden, gelb.
Entsteht jedoch eine heterocyclische Gruppierung (wo neben Kohlenstoff noch Sauerstoff als Ringglied fungiert), so tritt Farberhöhung auf, z. B.

$$
\mathrm{C}_{6} \mathrm{H}_{5} \cdot \underset{\mathrm{C}}{\mathrm{C}} \mathrm{C} \cdot \mathrm{C}_{6} \mathrm{H}_{5}
$$

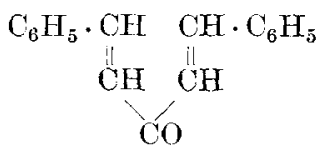

Diphenylpyron, farblos, Dibenzalaceton, gelb.

III. Farbigkeit und Konfiguration. Gibt der spezielle Bau der chromophoren Gruppe zur Bildung mehrerer stereoisomerer Formen Veranlassung, so treten, wie neuere Untersuchungen gezeigt haben, bei den beiden Stereomeren häufig Farbdifferenzen auf. So u. a. bei folgenden geometrisch-isomeren Athylenverbindungen, die stereoisomer sind im Sinne der Schemata:

$$
\begin{aligned}
& \mathrm{R} \cdot \mathrm{C} \cdot \mathrm{H} \quad \mathrm{Cis}-\quad \mathrm{R} \cdot \mathrm{C} \cdot \mathrm{H} \quad \text { Trans- } \\
& \mathrm{R}_{1} \cdot \stackrel{\text { C }}{\mathrm{C}} \mathrm{H} \text { Verbindung } \quad \mathrm{H} \cdot \stackrel{\mathrm{C}}{\mathrm{C}} \cdot \mathrm{R}_{1} \text { Verbindung }
\end{aligned}
$$

1. Diäthoxynaphthostilbene $\left.{ }^{19}\right) \mathrm{C}_{2} \mathrm{H}_{5} \mathrm{O} \cdot \mathrm{C}_{10} \mathrm{H}_{6} \cdot \mathrm{CH}$ : $\mathrm{CH} \cdot \mathrm{C}_{10} \mathrm{H}_{6} \cdot \mathrm{OC}_{2} \mathrm{H}_{5}$, höher schmelzend, labil, farblos; tiefer schmelzend, gelbe Tafeln.

2. Benzaldesoxybenzoine $\left.{ }^{20}\right) \quad \mathrm{C}_{6} \mathrm{H}_{5} \mathrm{CO} . \mathrm{CC}_{6} \mathrm{H}_{5}: \mathrm{CH}$ $\mathrm{C}_{6} \mathrm{H}_{5}$, höher schmelzend farblos; tiefer schmelzend gelb.

3. Dibenzoyläthylene'21) $\mathrm{C}_{6} \mathrm{H}_{5} \mathrm{CO} . \mathrm{CH}: \mathrm{CH} . \mathrm{COC}_{6} \mathrm{H}_{5}$, höher schmelzend farblos; tiefer schmelzend intensiv gelb.

18) Liebigs Ann. 349, 349.

9) EIbs, J. prakt. Chem. 4\%, 72

20) $\mathrm{S}$ t o b be u. $\mathrm{N}$ i ede $\mathrm{n} z \mathrm{u}$, Berl. Berichte 34, 3897.

21) Paa 1 u. Schulze, Berl. Berichte 33, $3795 ; 35,168$.

Auch bei verschiedenen geometrisch isomeren Diazoverbindungen ${ }^{22}$ ) sind derartige Differenzen aufgefunden, z. B.

4. $\quad \mathrm{C}_{6} \mathrm{H}_{5} \cdot \mathrm{N}$

$$
\mathrm{KSO}_{3} \cdot \mathrm{N}
$$

syn-Benzoldiazosulfonat, orange;

In letzterem Falle kommt dem labilen Isomeren, d. h. der Form mit höherem Energieinhalte, die die Tendenz hat, sich in die stabile Form umzuwandeln, die tiefere Farbe zu.

Zusammenfassend kann man den Satz aufstellen, daß die Chromophore, strukturchemisch gesprochen, durchwegs Doppelbindungen oder mehrfache Bindungen enthalten, d. h. ungesättigte Verbindungen darstellen, und daB an den Stellen der Doppelbindungen, den Lücken im Molekül, der Sitz für die Entstehung selektiver Absorption zu suchen ist ${ }^{23}$ ). Werden diese Doppelbindungen in einfache Bindungen übergeführt (z. B. durch Reduktion), d. h. die Lücken ausgefüllt, so verschwindet gleichzeitig die Farbigkeit; die farbigen ungesättigten Stoffe (Chromogene) gehen in die farblosen gesättigten Stoffe (Leukoverbindungen) über. Einige Beispiele mögen das belegen :

$$
\begin{aligned}
& \text { Chromogen: } \\
& \mathrm{C}_{6} \mathrm{H}_{5}-\mathrm{N}-\mathrm{N} \cdot \mathrm{C}_{6} \mathrm{H}_{5} \quad \mathrm{C}_{6} \mathrm{H}_{5} \mathrm{NH}-\mathrm{NHC}_{6} \mathrm{H}_{5} \\
& \mathrm{C}_{6} \mathrm{H}_{5}-\mathrm{N}: \mathrm{O} \\
& \mathrm{O}: \mathrm{C}_{6} \mathrm{H}_{4}: \mathrm{O} \\
& \mathrm{C}_{6} \mathrm{H}_{5} \mathrm{~N}: \mathrm{C}_{6} \mathrm{H}_{4}: \mathrm{Ne}_{6} \mathrm{H}_{5} \quad \mathrm{C}_{6} \mathrm{H}_{5} \mathrm{NH} \cdot \mathrm{C}_{6} \mathrm{H}_{4} \cdot \mathrm{NHC}_{6} \mathrm{H}_{5} \\
& \mathrm{C}_{6} \mathrm{H}_{5} \mathrm{CO} \cdot \mathrm{C} \cdot \mathrm{COO}_{6} \mathrm{H}_{5} \quad \mathrm{C}_{6} \mathrm{H}_{5} \mathrm{CO} \cdot \mathrm{C} \cdot \mathrm{COO}_{6} \mathrm{H}_{5} \\
& \text { O } \\
& \mathrm{OH} \mathrm{OH}
\end{aligned}
$$

IV. Farbänderung durch Einführung neuer Gruppen. Bathochromeund hy ps ochrome Gruppen. Auxochrome.

Sämtliche bisher besprochenen Chromophore enthaltenden Verbindungen, die man nach $W$ it $t$ als $\mathrm{Ch}$ rom og e n e bezeichnet, verändern durch Einführung bestimmter Radikale ihre Farbe, die bald erhöht, bald vertieft wird. Hier ist jedoch zu unterscheiden, ob durch Einführung der neuen Gruppe eine intramolekulare Umlagerung erfolgt, so daß die Farbänderung, die dann in der Regel diskontinuierlich verläuft, auf der Bildung eines ganz neuen, meist chinonähnlichen Chromophors beruht, oder ob die Einführung der neuen Gruppe einen weniger energischen Eingriff in das. Molekül des Chromogens bedeutet, und die Gruppe im wesentlichen nur durch ihre Anwesenheit, vielleicht rein physikalisch, indem die Sehwingungszahl geändert wird, die Verschiebung des Absorptionsbandes bewirkt. Im letzten Falle haben wir es mit der Wirkung von bathoohromen und hypsoc h r a m e n Gruppen zu tun. Von diesen verdienen die Amino- und Hydroxylgruppen eine besondere Erwähnung, die von Witt in seiner Farbstofftheorie

22) Hantzsch, Berl. Berichte $2 \%, 1702 \mathrm{ff}$.

23) Im Sinne der Auffassung J. J. Th o m s o n s über Valenz und Doppelbindung seheint auch eine plausible Deutung dieser Tatsachen möglich. 
zuerst als auxochrome Gruppen erkannt wurden, und die die Farbe des Chromogens vertiefen. Eine ähnliche Wirkung kommt den substituierten Gruppen, z. B. $\mathrm{N}\left(\mathrm{CH}_{3}\right)_{2}$ und $\mathrm{N}\left(\mathrm{C}_{2} \mathrm{H}_{5}\right)_{2}$ zu. AuBer der bloßen farbvertiefenden Wirkung beziehen bekanntlich diese Gruppen noch die den Farbchemiker interessierende Fähigkeit, das Chromogen in den Farbstoff zu verwandeln, d. h. eine Verbindung, die vermöge basischer oder saurer Eigenschaften Verwandtschaft zu Faserstoffen bekundet.

Als Beispiel, wo der Aminogruppe ein auxochromer Charakter zukommt, sei das braune Aminobenzol $\mathrm{NH}_{2} \cdot \mathrm{C}_{6} \mathrm{H}_{4} \mathrm{~N}_{-}^{-} \mathrm{N} \cdot \mathrm{C}_{6} \mathrm{H}_{5}$ erwähnt. Die Einführung der Aminogruppe in das orangefarbige Phenanthrenchinon läßt eine kupferrote Verbindung entstehen. Stark auxochrome Wirkungen scheint die Hydroxylgruppe im Tetraoxychinon zu entfalten :

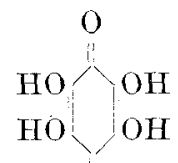

()

Besonders kräftig scheint die Wirkung der auxochromen Gruppen zu sein, falls sie mit dem Chromophor ringförmig verbunden sind, wie im Indigo ${ }^{24}$ ) :

$$
\mathrm{C}_{6} \mathrm{H}_{4}>\mathrm{NH}^{\mathrm{CO}}>\mathrm{C}_{-} \mathrm{CH}_{6} \mathrm{C}_{4},
$$

wo die Gruppen : - $-\mathrm{CO}-\mathrm{C}_{-}^{-} \mathrm{C}-\mathrm{CO}-$ die Rolle der Chromophore spielen; auch Acridon

$$
\mathrm{C}_{6} \mathrm{H}_{4}>\mathrm{CH}_{6} \mathrm{C}_{4}
$$

dürfte hier zu nennen sein.

Weiteres über gegenseitige Beziehungen zwischen Chromophor und Auxochrom siehe in der $\mathrm{Zu}$ sammenstellung bei $\mathrm{K}$ a u f f m a n $\mathrm{n}$ (S. 24-30).

Wie hervorgehoben werden soll, kann man von einer rein auxochromen Wirkung der Amino- und Hydroxylgruppe nur dann sprechen, wenn durch die Einführung dieser chemisch reaktiven Gruppen nicht gleichzeitig eine intramolekulare Umlagerung erfolgt, wie bei den Nitrophenolen. Derartige Umlagerungen sind aber auch in vielen anderen Fällen wahrscheinlich.

Von den übrigen Gruppen sei erwähnt, daß Alkyle und Aryle bathochrom wirken; bekanntlich ändert sich die Farbe mancher Farbstoffe, z. B. der Triphenylmethanreihe durch Einführung dieser Gruppen nach Blau; auch die $\mathrm{H}$ a loge n e scheinen häufig bathochrom $\mathrm{zu}$ wirken, während den $\mathrm{Acylen}$ z. B. $\mathrm{CH}_{3} \mathrm{CO}$ und $\mathrm{C}_{6} \mathrm{H}_{5} \mathrm{CO}$ in der Regel hypsochrome Eigenschaften zukommen.

In einigen Fällen entstehen auxochrome Gruppen durch gewisse chemische Eingriffe in Moleküle, die mehrere Chromophore enthalten, indem z. B. durch Reduktion aus dem einen Chromophor eine auxochrome Gruppe entsteht, während das andere Chromophor intakt bleibt. Nach R. S c h o l1 ${ }^{2 \bar{s}}$ ),

24) S c h o 11, Berl. Berichte 36, 3426 .

25) Berl. Berichte 36, 3426; 40,934. der diese Verhältnisse zuerst studierte, geht mit dieser Veränderung häufig eine Farbvertiefung des ursprünglichen Chromogens Hand in Hand; wie das Beispiel des

$$
\mathrm{CO}
$$

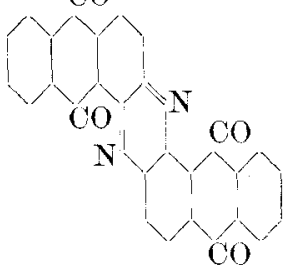

Anthrachinonazins, grüngelb

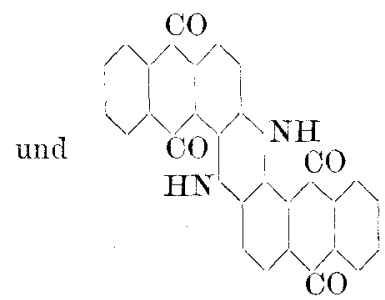

Indanthrens, blau lehrt, zeigt das Reduktionsprodukt Indanthren tiefere Farbe als sein Oxydationsprodukt, Anthrachinonazin; eine überraschende Erscheinung, die aber nach S o h o 11 durch folgende Betrachtung durchaus plausibel erscheint: Anthrachinonazin enthält drei Chromophore, die beiden Chinone und den orthochinoiden Azinchromophor. Wird es zu Indanthren reduziert, so bleiben erstere erhalten. und aus dem Azinchromophor gehen zwei stark auxochrome Aminogruppen hervor, die noch dazu an Ringbildung beteiligt sind. Es ist deshalb nicht einzusehen, weshalb mit einer solchen Veränderung eine Farberhöhung verbunden sein sollte.

V. Quantitative Beziehungen. Verschiebung der Absorptionsstrei. fen, Extinktionskoeffizient. Einf l u B der Lösungsmittel.

Um den Einfluß der bathochromen und hypsochromen Wirkung verschiedener Gruppen exakt festzustellen, ist es nötig, 1. die Verschiebung der Absorptionsstreifen im Spektrum des farbigen Stoffes und 2. die Stärke der Absorption in verschiedenen Gebieten zu messen. Die ersten Messungen, die zu stöchiometrischen Beziehungen geführt haben, sind von $\mathrm{G}$. $\mathbf{K} \mathbf{r} \ddot{\mathrm{u}} \mathrm{s}$ s an Derivaten des Indigos vorgenommen, die in chloroformischer Lösung einen Streifen im roten bis gelben Gebiete und starke Absorption im Grün aufweisen. Es zeigte sich, daß $\mathrm{CH}_{3}, \mathrm{OCH}_{3}, \mathrm{C}_{2} \mathrm{H}_{5}$ und $\mathrm{Br}$ den Streifen nach Rot, $\mathrm{NO}_{2}$ und $\mathrm{NH}_{2}$ nach Blau verschob. Spätere Messungen desselben Autors an bromierten und nitrierten Fluoresceinen ergaben das interessante additive Verhalten, daß die Einführung jedes Bromatoms in das Fluoresceïn dessen Absorptionsstreifen um nahezu 5,45 Wellenlängen, die Einführung jeder Nitrogruppe den Streifen um -1,3 Wellenlängen gegen Rot verschob. Auf spätere Messungen von B e r n th sen, E. K o c h, G. Kr ü s s und H. W. $\mathrm{V}$ o g e l sei hiermit verwiesen ${ }^{26}$ ). Ein additives Verhalten beobachtete kürzlich auch $H$. S t o b b e ${ }^{27}$ ) bei den Fulgiden in Chloroformlösung, wo die sukzessive Einführung bathochromer Phenylgruppen die Absorptionsbande um nahezu den gleichen Betrag nach dem roten Ende verschiebt.

26) Näheres's. Ostw ald, Lehrb. d. allg Chemie (I) 1903, 464; ferner $\mathrm{K}$ a y s e r, Spektroskopie.

27) Liebigs Ann. 349, 364. 
Zur erschöpfenden Definition des Begriffes der Farbigkeit ist ferner nötig, die $\mathrm{F}$ a r binte $\mathrm{n}$. sität, d. h. die $S t a ̈ r k e d e r A b s o r p t i o n$ in verschiedenen Absorptionsgebieten zu bestimmen, was bekanntlich durch Messung des Extink tionskoeffizienten E geschieht, worunter man den reziproken Wert der Schichtdicke versteht, die nötig ist, um das durchfallende Licht auf ein Zehntel der Intensität des auffallenden Lichtes abzuschwächen. Je größer somit $\mathrm{E}$ ist, desto größer ist die Farbintensität. Beschränken wir uns auf Lösungen der absorbierenden Stoffe, so ist bekannt, daß fast allgemein die Stärke der Absorption mit der Konzentration $\mathrm{C}$ der Lösung wächst. Für viele Stoffe ist das sogen. B e e r sche Gesetz gültig, nach $\operatorname{dem} \mathrm{E} / \mathrm{e}=\mathrm{A}$ (Absorptionsverhältnis), d. h. für verschiedene Konzentrationen das Absorptionsverhältnis konstant ist. Nichtkonstanz des Absorptionsverhältnisses läßt in der Regel auf eine mit wechselnder Konzentration vor sich gehende Veränderung des gelösten Stoffes schließen ${ }^{28}$ ).

Messungen ron Extinktionskoeffizienten bei konstitutiv unveränderlichen Stoffen sind neuerdings von $\mathrm{Hantzsch}$ und $\mathrm{Glover}{ }^{29}$ ) angestellt; es gelangten Lösungen von Bisdiphenylenäthen, Azobenzol, Oxyazobenzol und Substitutionsprodukte RO. $\mathrm{C}_{6} \mathrm{H}_{4} \cdot \mathrm{N}_{2} \cdot \mathrm{C}_{6} \mathrm{H}_{5}$, Benzochinon und Chi. nonoxime zur Unter uchung. Für die Lösungen dieser Stoffe erwies sich das B e e r sche Gesetz bis zu stiarken Verdünnungen hinauf als gültig.

EinfluB der Lösungsmittel. Bei ciesen Untersuchungen ist man fast durchweg auf die Benutzung von Lösungsmitteln angewiesen, wodurch sofort als komplizierender Faktor die Tatsache hinzutritt, daß die Absorptionsspektra auch von der Natur der Lösungsmittel mehr oder weniger abhängig sind. In manchen Fällen gilt hier die sogen. K u n d $\mathbf{t}$ sche Regel, nach der sich bei den verschie denen Lösungen die Absorptionsstreifen um so mehr gegen das rote Ende verschieben, je stärker das Lösungsmittel für den betr. Absorptionsbereich dispergiert ${ }^{30}$ ). Diese Regel hat jedoch, wie noch neuerdings) wiederholt konstatiert wurde, durchaus keine allgemeine Gültigkeit. Die Ausnahmen werden vielleicht $z . T$, dadurch bedingt, daß Lösungsmittel und gelöster Stoff chemisch aufeinander wirken, indem etwa Verbindungen zwischen beiden entstehen, oder das Lösungsmittel umlagernd wirkt.

Von neueren Beispielen, die starken Einfluß des Lösungsmittels auf die Absorption dartun, sei das von $K$ a uff $m$ a $\mathbf{n ~ n}^{31}$ ) qualitativ studierte Beispiel des 3-Aminophthalimids:

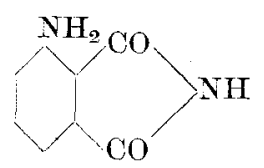

angeführt, das in verschiedenen Lösungsmitteln verschiedene Farbintensität und auch wechselnde Fluorescenz besitzt.

28) Siehe z. B. B a u r, Spektroskopie.

29) Berl. Berichte 39, 4153.

s0) Näheres s. K a y s e r, Handb. III, 80ff.

31) Siehe z. B. K a uf $\mathrm{m}$ a $n \mathrm{n}, \mathrm{Z}$. physikal. Chem.
Sehr erheblich ist der Einfluß des Lösungsmittel auf die von $H$ ant $\mathrm{zsch}$ und $\mathrm{G}$ lover in ihrer oben zitierten Arbeit untersuchten Stoffe. So wurden für Azobenzol (Thalliumlicht und $1 / 800$ normale Lösungen) folgende Extinktionskoeffizienten $\mathrm{E}$ gefunden :

$\begin{array}{ccccc}\text { Lösgm. } & \text { Benzol } & \text { Ligroin } & \text { Chloroform } & \text { Ather } \\ \text { E } & 0.0363 & 0.0342 & 0.0316 & 0.0304 \\ \text { Aceton } & \text { Alkohol } & \text { Eisessig } & \text { Alkohol }+50 \% \mathrm{H}_{2} \mathrm{O} \\ 0.0226 & 0.0275 & 0.0269 & 0.0193\end{array}$

Sauerstoffhaltige Lösungsmittel vom Typus des Wassers wirken somit sehr viel stärker farbaufhellend als Kohlenwasserstoffe wie Benzol, wahr. scheinlich deshalb, weil erstere Lösungsmittel mit den gelösten Azokörpern Verbindungen von hellerer Farbe erzeugen.

Im entgegengesetzten Sinne beeinflussen die Lösungsmittel die Farbintensität der Lösungen des Nitrohydrochinondimethylesters. Wie zuerst von $\mathrm{K}$ a $\mathrm{u}$ f f $\mathbf{m}$ a $\mathbf{n} \mathbf{n}^{\mathbf{3}}$ ) nachgewiesen wurde, besitzt dieser Nitrokörper von anscheinend unveränderlicher Konstitution im festen Zustande intensiv gelbe Farbe; auch dissoziierende Lösungsmittel, wie Wasser, Alkohole, Eisessig, lösen mit mehr oder weniger gelber Farbe, während sich der Ester in indifferenten Lösungsmitteln, wie Ligroin, Hexan usw., farblos löst. Eine genaue quantitative Untersuchung dieses Falles von $\mathrm{H}$ a $n \mathrm{t} \mathrm{z} \mathrm{c} \mathrm{h}^{33}$ ) erbrachte den Beweis, daß die rend. Hexanlösung des Nitrohydrochinondimethylesters, die fast völlig farblos ist, den Ester in monomolekularem Zustande enthält, in konz. Lösungen nimmt die Molekulargröße zu und gleichzeitig erlangen die Lösungen schwach gelbe Farbe. Auch die intensiv gelben methylalkoholischen Lösungen enthalten den Ather in monomolekularem Zustande; jedoch wächst hier das Molekulargewicht nicht mit steigender Konzentration, was wohl so $\mathrm{zu}$ deuten ist, daß in der methylalkoholischen Lösung gelbe Assoziationsprodukte des monomolekularen Nitroäthers enthalten sind. Eine Untersuchung der Extinktionskoeffizienten der verschiedenen Lösungen ergab, daß auch in scheinbar farblosen Aethylacetatlösungen, wo der Ester mono molekuler ist, - jedenfalls infolge Bildung von farbigen Polymeren - das B e e r sche Gesetz nicht streng gilt. Somit enthalten derartige verdünnte farblose Lösungen den an sich farblosen Nitroäther, d. h. in normalem Zustande und nicht, wie Kauffmann meint, in einem Zwangszustande; die gelbe Farbe der alkoholischen Lösungen ist vielleicht auf Bildung von Assoziationsprodukten mit dem Lösungsmittel, z. B. $\mathrm{CH}_{2} \mathrm{OH}$, zurückzuführen, während die gelbe Farbe der konz. Hexanlösungen, was die Molekulargewichtsbestimmungen direkt wahrscheinlich machen - auf Bildung von Polymerisationsprodukten beruht, etwa im Sinne folgender, nicht strukturell gedachter Formeln :

$$
\begin{gathered}
\mathrm{C}_{6} \mathrm{H}_{3}\left(\mathrm{OCH}_{3}\right)_{2} \text { farblos, } \\
\left.\mathrm{C}_{6} \mathrm{H}_{3} \mathrm{OCH}_{3}\right)_{2} \ldots\left(\mathrm{CH}_{3} \cdot \mathrm{OH}\right)_{n} \text { gelb, } \\
\left.\mathrm{NOH}_{3}\right)_{2} \ldots \mathrm{NO}_{2} \backslash \mathrm{C}_{6} \mathrm{H}_{3} \text { gelb. } \\
\mathrm{OO}_{2} \ldots\left(\mathrm{CH}_{3} \mathrm{O}\right)_{2}
\end{gathered}
$$

32)Berl. Berichte 39, 4237.

33) Berl. Berichte 40, 1556. 
Daß die farblosen Lösungen den Stoff in normalem Zustande enthalten, wird auch unter Berücksichtigung der Tatsache sehr wahrscheinlich, daß die Molekularrefraktion des Nitrohydrochinonäthers in den farblosen Lösung den kleinsten d. i. normalsten, in der gelben alkoholischen Lösung den größten Wert aufweist.

Von Interesse sind ferner Beziehungen zwischen der dielektrischen Kraft der Lösungsmittel und ihrer farberteilenden Fähigkeit, auf die früher von $K$ a $u f f m$ a $n n^{34}$ ) bei anderer Gelegenheit schon aufmerksam gemacht hat, und auf die hiermit nur verwiesen werden kann.

\section{Erweiterung der Auxoehrom- theorie.}

Bei dem heutigen Stande der Strukturchemie scheint die in deren Grundzügen skizzierte Auxochromtheorie, speziell bei konstitutiv unveränderlichen Verbindungen, die einfachste Darstellungsform der beobachteten Tatsachen zuzulassen; bei konstitutiv veränderlichen, z. B. tautomer reagierenden Stoffen wird die Auxochromtheorie in ihrer ursprünglichen Form in manchen Fällen der besonders von $\mathrm{H}$ a $\mathrm{n} t \mathrm{z} \mathrm{s} \mathrm{ch}$ entwickelten Umlagerungstheorie Platz machen müssen, die im Kap. VII genauer besprochen wird. Aber auch bei nicht tautomerisierbaren Gebilden bleibt die Auxochromtheorie die Antwort auf viele Fragen schuldig. Fitra $g$ hört $z$. B. die Tatsache, daß das deutlich gello Nitronaphthalin durch Einführung einar zweiten Nitrogruppe, also nach bisheriger Anschanung eines farbvertiefenden Eiementes, fast farbios wira. Es ist deshalb von Bedeutung, daB H. K a u f $\mathrm{f}$ mann eine Erweiterung der Auxochromtheorio angestrebt hat, speziell bei solchen Verbindungen, die Auxochrom und Chromophor in Verbindung mit einem Benzolring enthalten, und dic, wie bekannt, in dieser Vereinigung besonders häufig zur Farbig. keit Veranlassung geben. Es liegt nun, besonders mit Rücksicht auf die Untersuchungen $A$. $v$. $B$ a e y e r s über die Konstitution des Benzols die Annahme nahe, daß der Benzolring bei Einführung gewisser Gruppen seinen Zustand ändert:

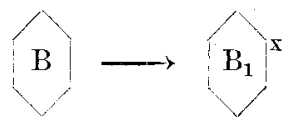

und daß mit dieser Änderung auch eine solche der Lichtabsorption verbunden ist, eine Annahme, die diskutabel erscheint, wenn man berücksichtigt, daB Benzol an sich ,farbig“" ist. Anhaltspunkte für diese Anschauung von verschiedenen, ,Zuständen des Benzolringes" gaben Untersuchungen über das Leuchten von Benzolderivaten unter dem Einfluß von Teslaströmen und Radiumstrahlen, die von $\mathrm{K}$ a u f f $\mathrm{man} \mathbf{n}^{3-1}$ ) angestellt sind, und die $\mathrm{u}$. a. folgende Resultate gezeitigt haben :

1. Dämpfe farbiger Benzolderivate leuchten nicht.

34) Z. physikal. Chem. 50, 350. Berl. Berichte 3\%, 2941
2. Auxochrome rufen Leuchten hervor oder verstärken dasselbe je nach Maßgabe der auxo. chromen Eigenschaft.

3. Chromophore wirken dem Leuchten entgegen.

Parallel mit der Eigenschaft zu luminescieren geht das magneto-optische Verhalten der Stoffe, das hier durch die sogen. magneto-optische Anomalie gemessen wird, d. h. die Differenz zwischen der beobachteten und berechneten magnetischen Molekularrotation (P e rkin). Stoffe, die hohe Werte der magneto-optischen Anomalie aufweisen, haben die Eigenschaft zu luminescieren und im allgemeinen auch zu fluorescieren. Die Zustandsänderung des Benzolringes wird mit den Hilfsmitteln der Strukturchemie, am einfachsten durch eine Lagenänderung der einfachen und doppelten Bindungen, zum Ausdruck gebracht. In Anlehnung an A. v. B a e y e $r$ unterscheidet $\mathbf{K}$ a $\mathrm{u}$ f f $\mathrm{m}$ a $\mathrm{n} \mathbf{n}$ für das Benzol drei Grenzzustände: 1. den durch die Diagonalformel zum Ausdruck gebrachten, 2. den $\mathrm{K} \mathrm{e} \mathrm{k}$ u 1 é schen, 3. den $\mathrm{D}$ e w a r schen ${ }^{35}$ ):
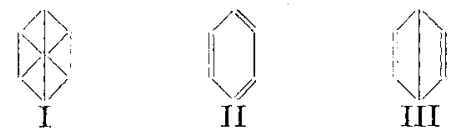

Im Zustande I, der den aliphatischen Charakter symbolisieren soll, befinden sich Stoffe mit stark negativen Substituenten wio $\mathrm{C}_{6} \mathrm{H}_{4}\left(\mathrm{NO}_{2}\right) \mathrm{CO}_{2} \mathrm{H}$. $\mathrm{Da}$ hier eigentliche Doppelbindungen fehlen, so wirkt dieser Zustand auch für die Farbgebung ungünstig. Der zweite Grenzzustand, den aromatischen Charakter darstellend, ist z. B. in den Phenolen ijxuert. Ua in diesem die Doppelbindungen am dich. testen verteilt sind, so ist dieser Zustand besonders zur Hervorrufung von Farbe geeignet. Im dritten Grenzzustande, der der D e w a s schen Formel entspricht, befindet sich der Benzolring z. B. im Anilin, p-Phenvlendiamin usw., anch scheinbar in einigen Kohlenwasserstoffen wie Naphthalin und Anthracen, d. h. Stoffen, die zur Bildung chinonartiger Stoffe (z. B. durch Oxydationsreaktionen) prädestiniert sind. Da die Zahl der Doppelbindungen geringer ist als bei $\mathrm{Ii}$, so wird anch das Aufkommen von Farbe weniger begünstigt als im zweiten Grenz. zustande. Nach $K$ a u f f $m$ a $n \mathbf{n}$ ist der $D$ e w a $r$ sche Zustand dadurch nachweisbar, daß die Dämpfe der in diesem Zustande befindlichen Stoffe unter dem Einfluß von Teslaströmen violett leuchten, und die Stoffe gleichzeitig hohe Werte der magnetooptischen Anomalie aufweisen.

Denken wir uns nun irgend eine Benzolverbindung, die sich je nach der Natur der chromogenen Gruppe in einem der drei Zustände oder, allgemeiner, in einem dazwischenliegenden Zustande befindet, so wird nach Einführung einer neuen Gruppe der Benzolring als empfindliches Gebilde mit einer Verschiebung seines jeweiligen Zustande reagieren. Auxochrome Gruppen sind dadurch charakterisiert, daß sie den Zustand eines Benzolringes derart verändern, daß ein Maximum von Doppelbindungen erzielt wird. Nach der Stärke ihrer Wirkungen teilt $K$ a $u$ f $f m$ a $n$ die Gruppen in folgende Reihe ein :

35) Berl. Berichte 33, 1725; 34, 682; 35, 3668; Z. physikal. Chem. 55, 547 . 


$\begin{array}{cccc}\mathrm{OCOCH}_{3} & \mathrm{OCH}_{3} & \mathrm{NHCOCH}_{3} & \mathrm{NH}_{2} \\ -0,26 & -1-1,46 & 1,95 & 8,82 \\ & \mathrm{C}\left(\mathrm{CH}_{3}\right)_{2} & \mathrm{~N}_{2}\left(\mathrm{C}_{2} \mathrm{H}_{5}\right)_{2} & \\ & 8,59 & 8,82 & \end{array}$

Die zustandsverschiebende Wirkung der Alkoxylgruppen ist somit nicht bedeutend, während die zweifach substituierten Aminogruppen die stärksten Wirkungen äußern. Die Zahlen bedeuten die an den betreffenden Benzolderivaten $\mathrm{C}_{6} \mathrm{H}_{5} \mathrm{OCOCH}_{3}$ $\mathrm{C}_{6} \mathrm{H}_{5} \mathrm{OCH}_{3}$ usw. gemessenen Zahlen der magnetooptischen Anomalie.

Angliederung von Ringen an den Benzolkern übt denselben farbvertiefenden Einfluß aus wie Auxochrome, was z. B. direkt aus den Zahlen der magneto-optischen Anomalie folgt, deshalb geben z. B. Diphenyl nahezu farblose, die böher molekularen Kohlenwasserstoffe, wie Pyren und Chrysen gelbe Nitrokörper. Die gelbe Farbe der Nitraniline erklärt K a u f f m a n $n$ folgendermaßen : im Nitrobenzol ist der Ring in einem der Diagonalformel nahezu entsprechenden Zustande, die Einführung der Aminogruppe bewirkt sehr weitgehende Annäherung an den durch die folgende Formel :

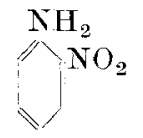

dargestellten Zustand, mit dem Farbigkeit verbunden ist.

Von Einfluß auf die Verschiebung des Benzolzustandes ist ferner die Stellung der Auxochrome untereinander, was $\mathrm{K}$ a uff $\mathrm{mann}{ }^{36}$ ) in seinem sog. Verteilungssatzder A uxochrome zum Ausdruck bringt. Die Auxochrome (in den folgenden Beispielen die $\mathrm{OCH}_{3}$-Gruppen) unterstützen sich in p-Stellung: Hydrochinondimethyläther (I)<smiles>CO[N+]1([O-])CCCCC1C</smiles><smiles>COC1CCCCC1(OC)OC</smiles>

ist stark gelb (im festen Zustande). Nitroresorcindimethyläther (II) gelblichweiß. Außerdem ist die Art des Chromophors von EinfluB. 4-Nitroveratrol (III) ist blaßgelb<smiles>COC1CCC(OC)OC1</smiles><smiles>COC1CCC(=O)C(OC)O1</smiles>

3-Nitroveratrol (IV) fast farblos. Für das Zustandekommen der Farbe treten nach $\mathbf{K}$ a u f $\mathrm{fman} \mathbf{n}$ dieselben begünstigenden und hindernden Einflüsse zutage wie bei der Fluorescenz $z^{37}$ ).

Als sehr stark auxochrom wirkende Gruppe, die den Zustand des Benzols sehr weitgehend verschieben soll, sieht $\mathrm{K}$ a u $\mathrm{f} f \mathrm{~m}$ a $\mathrm{n}$ die Gruppe $\mathrm{OMe}$, z. B. ONa an und erklärt so die intensive Farbe der Nitrophenolsalze, wobei zugleich der Versuch gemacht wird, die Theorie der Partialvalenzen für

36) Berl. Berichte 39, 2722.

37) Näheres s. Kauffmann, Fluorescenz und chemische Konstitution, Ahrens' Sammlung Bd. XI.

den vorliegenden $Z$ weck zu verwerten. Hiermit gelangen wir aber schon in das Gebiet der konstitutiv veränderlichen Verbindungen, wo die im nächsten Kapitel zu entwickelnde Umlagerungstheorie von $\mathrm{H}$ a $\mathbf{n ~ t ~ z ~ s ~ c ~ h ~ i m ~ G e g e n s a t z ~ z u ~ K ~ a ~ u ~ f ~ f - ~}$ manns Theorie befriedigendere Erklärung der Erscheinungen zuläßt.

VII. Umlagerungstheorie. Farbänderungen bei der Salzbildung.

Im Gegensatz zu den bisher besprochenen Erscheinungen stehen Farbänderungen, meist Farb. vertiefungen, die häufig bei der Salzbildung umlagerungsfähiger, $d, h$. konstitutiv veränderlicher Stoffe beobachtet und besonders von $\mathrm{Han} \mathrm{tz} \mathrm{s} \mathrm{h}$ 38) im Anschluß an seine Untersuchungen über Pseudosäuren studiert worden sind.

Pseudosäuren sind bekanntlich Wasserstoffverbindungen, die bei der Salzbildung eine Umlagerung erleiden, deren Salze sich somit von einer anderen, stärker saueren, Form (aci-Form) ableiten. In einigen Fällen, wie beim Phenylnitromethan, ist die echte Säure auch als solche isoliert, in anderen, wie an gewissen aliphatischen Dinitro-Kohlenwasserstoffen, läßt sich ihre vorübergehende Existenz mit aller Schärfe nachweisen ${ }^{33}$ ). Wir haben also:

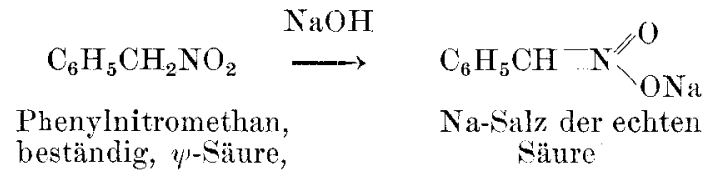

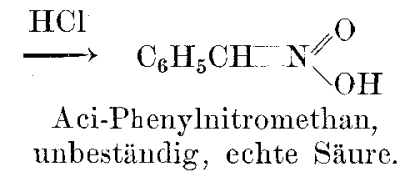

In einigen Fällen ist zugleich mit der unter Umlagerung verlaufenden Salzbildung A u f $\mathrm{tre}$ te $\mathrm{n}$ v on Farbe verbunden, wie beim Dinitroäthan und anderen Dinitroverbindungen :

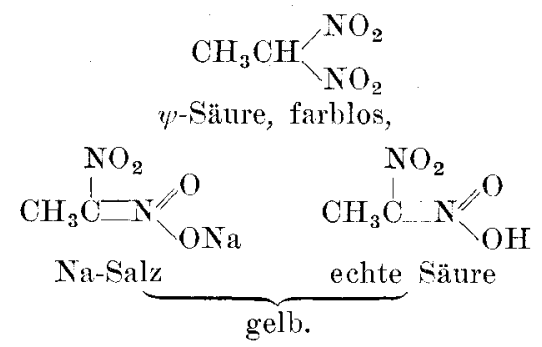

Im Gegensatz zu den $y^{\prime}$-Säuren, die in der Reihe der aliphatischen Nitro- und Dinitroverbindungen an sich völlig neutrale Stoffe darstellen, sind die aciNitroverbindungen Elektrolyte, deren Alkalisalze z. B. das oben formulierte Dinitroäthannatrium in wässeriger Lösung normaler Weise elektrolytisch dissoziiert sind. In wässeriger Lösung derartiger $\psi$-Säuren ist häufig ein deutlich nachweisbarer Gleichgewichtszustand vorhanden (Ley und Hantzsch) Berl. Ber. 39, 41:

38) Berl. Berichte 32, 575 .

39) Hantzsch u. Veit, Berl. Berichte 33, 626; Ley u. Hantzsch, Berl. Berichte 39, 3149 . 


$$
\begin{aligned}
& \mathrm{CH}_{3} \mathrm{OH}\left(\mathrm{NO}_{2}\right)_{2} \stackrel{\mathrm{I}}{\rightleftarrows} \mathrm{CH}_{3} \mathrm{C}\left(\mathrm{NO}_{2}\right) \mathrm{NO} \cdot \mathrm{OH} \\
& \rightleftarrows \mathrm{CH}_{3} \mathrm{C}\left(\mathrm{NO}_{2}\right) \mathrm{NO}^{\prime} \mathrm{O}^{\prime}+\mathrm{H}^{-},
\end{aligned}
$$

der dadurch charakterisiert ist, daß I. die Pseudosäure mit der echten Säure und letztere wieder mit den Ionen der echten Säure im Gleichgewicht ist. II.

Ahnliches scheint bei den Nitrolsäuren der Fall zu sein, die als farblose Wasserstoffverbindungen rote Salze liofern, denen wohl folgende Konstitution zukommt :

$$
\mathrm{R} \cdot \mathrm{O} / \mathrm{NO}_{2}
$$

Nitrolsäure, farblos, $\psi$-Säure

und die in farblose isomere Salze übergehen.

Bei dem thergange der farblosen Oximidoketone, Verbindungen der Gruppe - $\mathrm{COC}(\mathrm{NOH})$ in die farbigen (gelben) Salze, ist gleichfalls eine Atomverschiebung die Ursache der Farbigkeit.

Sehr auffällig ist ferner die Umwandlung der farblosen Violursäure :<smiles>O=C1NC(=O)C(=O)N1</smiles>

in die tief violettroten Alkalisalze.

$\mathrm{Hantzsch}$ betrachtet ganz allgomoin als Charakteristikum einer Pseudosäure die Tatsache, daß eine in dissoziierenden Solvenzien farblos lösliche Wasserstoffverbindung farbige Ionen und farbige feste Alkalisalze bildet oder noch allgemeiner jedes Auftreten oder jede Veränderung von Körperfarbe bei der Bildung von Salzen mit farb. losen Metallatomen ist a u intramolekulare Umlagerung zurückzu führen.

a) Auftreten von Farbe bei Salzbildung der Nitrophenole. (Indicatortheorie.)

Die bei der Salzbildung der aliphatischen Dinitroverbindungen auftretenden Farbänderungen erinnern auffällig an analoge Erscheinungen bei den Nitrophenolen und beweisen, daß auch hier dio Salzbildung von einer intramolekularen Umlagerung begleitet ist. Die Entstehung der Farbe ist also hier nicht auf die Gegenwart der chromogenen $\mathrm{NO}_{2-}$ und auxochromen OH-Gruppe zurückzuführen, sondern darauf, daß sich eine neue chinoide Atomgruppierung bildet, die vermöge ihres mehr ungesättigten Charakters Farbigkeit erzeugt. DaB die roten Alkalisalze der Nitrophenole dieselbe Konstitution wie die Wasserstoffverbindungen besitzen sollen, ist schon aus dem Grunde unwahrscheinlich, daß bei konstitutiv unveränderlichen Wasserstoffverbindungen der bloße Ersatz des Wasserstoffs durch Alkalimetalle niemals genügt, um eine tiefgreifende Farbveränderung hervorzurufen. Ein strenger Beweis für die Pseudosäurenatur der Nitrophenole liegt darin, daß es $\mathbf{H}$ ant $\mathrm{z}$ s eh und G o r k e ${ }^{40}$ ) gelang, zwei isomere Äther der Nitrophenole darzustellen. Neben dem schon längst be- kannten stabilen in reinem Zustande $\mathrm{f}$ a $\mathrm{r}$ b l o s e $\mathrm{n}$ Ather existiert noch ein labiler roter Ather. Ersterer ist der wahre Nitrophenoläther, letzterer leitet sich von der chinoiden Form des Nitrophenols ab und ist als chromo-Nitrophenol. äther zu bezeichnen :<smiles>COc1ccc([N+](=O)[O-])cc1</smiles>

echter Nitrophenoläther, Benzolderivat, sehr schwach gelb.<smiles>CO[Si](=O)C1C=CC(=O)C=C1</smiles>

chromo-Nitrophenoläther, Chinonderivat, tief rot.
Bezeichnet man das Nitrophenol allgemein mit

$$
\mathrm{Ar}_{\mathrm{OH}}^{\mathrm{NO}}
$$

so ist der wahre (benzoide) Nitrophenoläther als

$$
\mathrm{Ar}_{\mathrm{OO}} \mathrm{NO}_{2 n+1}
$$

der (chinoide) chromo-Nitrophenoläther als

$$
\mathrm{Ar}_{\mathrm{O}}^{\mathrm{NO} \cdot \mathrm{OC}_{n} \mathrm{H}_{2 n}+1}
$$

zu formulieren. Derartige labile chromo-Äther sind bei der Pikrinsäure, 2,4-Dinitrophenol, o-Nitrophenol aufgefunden und sind auch bei anderen Nitrophenolen sowie Nitronaphtholen angedeutet, sie sind als Verbindungen mit höherem Energieinhalt labil und haben die Tendenz, sich in die farblosen echten Nitrophenoläther umzulagern. Diese Beobachtungen werden dahin verallgemeinert, daß alle Nitrokohlenwasserstoffe der Fettreihe wie der Benzolreihe sowie alle Polynitroverbindungen und alle substituierten $\mathrm{Nitrobenzole} \mathrm{mit} \mathrm{kon}$ stitutivunveränderlichen Substi tucnten, z. B.

$$
\mathrm{C}_{6} \mathrm{H}_{4} \mathrm{NO}_{2} \mathrm{OCH}_{3}, \quad \mathrm{C}_{6} \mathrm{H}_{3} \overbrace{\left(\mathrm{NO}_{2}\right)_{2}}^{O \mathrm{OH}_{3}}, \mathrm{C}_{6} \mathrm{H}_{2} \underbrace{\mathrm{OCH}_{3}}_{\left(\mathrm{NO}_{2}\right)_{3}} \text {. }
$$

f a $r$ b 1 os sind. Die Nitrogruppe ist also in allen diesen Verbindungen nicht als Chromophor zu betrachten.

Die Farbe der freien festen Nitro. $\mathrm{phenole}$ ist verschieden, in einigen Fällen, wie beim p-Nitrophenol, 2, 4-Dinitrophenol, sind die Verbindungen farblos; somit liegen die den farblosen Estern entsprechenden wahren (benzoiden) Nitrophenole vor :

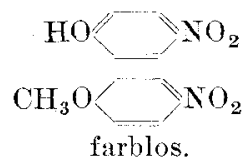

In anderen Fällen sind die freien Wasserstoffverbindungen gelb wia beim o-Nitrophenol; alsdann erscheint es plausibel, die festen Stoffe als homogene feste Lösungen der chromo-Form in der wahren (farblosen) Nitrophenolform aufzufassen, d. h. ein Gleichgewicht zu supponieren, das zum Teil dem früher erwähnten in den Lösungen gewisser $\mathrm{p}$-Säuren

4) Berl. Berichte 39, 1073. 
vorhandenen an die Seite zu stellen ist. Durch diese Auffassung wird manche Tatsache sofort verständlich, für die die alte Chromophortheorie keine Erklärung hatte, so z. B. die Farblosigkeit des Dinitrophenols im Gegensatz zu dem gelben o-Nitrophenol. Wenn nämlich die Körperfarbe erst durch Umlagerung zustande kommt, so ist es begreiflich, daß die Umlagerungstendenz:

$$
\mathrm{Ar}_{\mathrm{OH}}^{\mathrm{NO}} \longrightarrow \mathrm{Ar}_{\mathrm{O}}^{\mathrm{NO} \cdot \mathrm{OH}}
$$

durch jecle konstitutive Änderung sowohl gesteigert als auch geschwächt werden kann.

Modifizierung der Indicatortheorie.

Die Entdeckung der chromo-Nitrophenolester istnun fundamentalfür die Auffasssung der Konstitution der Njtrophenolsalze geworden. Einigen roten chromo-Nitrophenolestern entsprechen auch Alkalisalze von genau gleicher Farbe, die in einigen Fällen. so beim o-Nitrophenolkalium schon seit längerer Zeit bekannt sind :
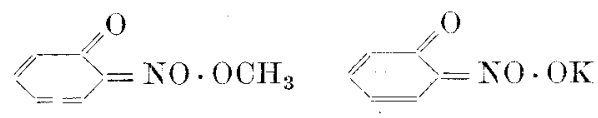

Bekanntlich sind auch die wäsesrigen Lösungen der Nitrophenolsalze intensiv farbig, was die frühere Indicat ortheorie durch die Bildung eines farbigen Nitrophenolanions zu erklären versuchte. Die Existenz der elektrisch neutralen chromo-Nitrophenolester beweist aber, daß diose Theorie nicht richtig sein kann, es ist nicht die Ionenbildung die primäre Ursache der Farbigkeit, sondern die mit der Substitution des stark elektropositiven Alkalimetalles erfolgende und durch die obigen Formulierugnen zum Ausdruck gebrachte Umlagerung. Durch diese Untersuchungen ist somit auch die rein chemische Theorie der Indicatoren wieder in ihre Rechte eingesetzt ${ }^{41}$ ).

Eine ähnliche Auffassung ist schon früher von Vorländer ${ }^{42}$ ) bei Gelegenheit der Farbänderung des Aminoazobenzols durch Säuren geltend gemacht worden. Wäre die frühere Ansicht richtig, nach der die intensiv violettrote Farbe der sauren I.ösungen des Aminoazobenzols lediglich auf Salzbildung, d. h. auf Bildung des Ions $\left(\mathrm{NH}_{3} \cdot \mathrm{C}_{6} \mathrm{H}_{4} \cdot \mathrm{N}\right.$ : $\mathrm{NC}_{6} \mathrm{H}_{5}$ ) beruhe, so sollte auch das Ion des Trimethylammoniumazobenzols $\left[\mathrm{N}\left(\mathrm{CH}_{3}\right)_{3} \mathrm{C}_{6} \mathrm{H}_{4} \cdot \mathrm{N}: \mathrm{N}\right.$ . $\mathrm{C}_{6} \mathrm{H}_{5}$. [ [entsprechend dem Salze $\mathrm{ClN}\left(\mathrm{CH}_{3}\right)_{3} \mathrm{C}_{6} \mathrm{H}_{4} \cdot \mathrm{N}_{2}$ . $\left.\mathrm{C}_{6} \mathrm{H}_{5}\right]$ von ähnlicher intensiver Farbe sein. Tatsächlich sind aber die Salze des Trimethylammoniumazobenzols kaum anders farbig als Azobenzol; mit der Ionenbildung kann also die Farbe nicht in direktem Zusammenhang stehen. Höchstwahrscheinlich wird auch hier bei der Salzbildung eine Umlagerung in eine weniger gesättigte (chinoide) Form erfolgen.

Nach einer neueren Untersuchung von $\mathrm{H}$ a n $\mathrm{tzsch} \mathrm{c}^{43}$ ) sind die Verhältnisse bei der Salzbildung der Nitrophenole aber insofern komplizierter, als außer den erwähnten roten Salzen di-

41) Hantzsch, Berl. Berichte 39, 1089; s. a. I a r g o s ches, diese $Z$., woselbst auch weitcre Lit. ïber die Indicatoren zu finden ist.

42) Berl. Berichte 36, 1485; vgl. Liebigs Ann. 320,116

$$
\text { 43) Ber]. Berichte 40, } 330 \text {. }
$$

verse isomere g e 1 b e Salze existieren. Die Struktur dieesr Salze ist noch nicht mit Sicherheit aufgeklärt, vielleicht ist die Isomerie sterischer Natur und der der roten und gelben Diazosulfonate (S. 1308) vergleichbar :

$$
\begin{array}{cc}
\mathrm{Ar}-\mathrm{N} & \mathrm{Ar}-\mathrm{N} \\
\mathrm{MeSO} \cdot \mathrm{N} & \mathrm{N} \cdot \mathrm{SO}_{3} \mathrm{Me} \\
\text { syn-Salz, } & \text { anti-Salz, } \\
\text { dunkler farbig, } & \text { heller farbig, } \\
\mathrm{C}_{6} \mathrm{H}_{4}-\mathrm{O} & \mathrm{C}_{6} \mathrm{H}_{4}-\mathrm{O} \\
\mathrm{NO} & \mathrm{MeO} \cdot \mathrm{ON} \\
\text { syn-Salz, rot, } & \text { anti-Salz, gelb. }
\end{array}
$$

Zur einfacheren Darstellung dieser Verhältnisse mit Hilfe von Strukturformeln ist für das chromo-Nitrophenol die "Superoxydformel" des Chinons zu

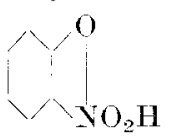

Grunde gelegt : z. B.

Außer diesen auch krystallographisch verschiedenen isostereomeren Formen treten bei den Nitrophenolen häufig primär noch orangefarbige Alkalisalze auf, die als "Mischsalze" d. h. als Gemenge resp. feste Lösungen oder als lockere chemische Verbindungen der gelben und roten Formen anzusehen sind, und die auch bisweilen durch fraktionierte Krystallisation in die Komponenten zerlegt werden können.

Am eingehendsten ist die Salzbildung beim Tribrom-m-dinitrophenol :<smiles>O=C1O[W](=O)C(Br)=C1Br</smiles>

untersucht. Dieser völlig farblose Nitrokörper bildet primär ein orangefarbiges Mischsalz

$$
\mathrm{C}_{6} \mathrm{Br}_{3} \mathrm{NO}_{2} \mathrm{NO}_{2} \mathrm{~K} \text {, }
$$

das sich auch bei tiefen Temperaturen bildet und durch wasserfreie Lösungsmittel in die Komponenten, das labile gelbe und das weniger labile rote Salz zerlegen läBt. Daß in letzteren beiden Salzen labile Verbindungen vorliegen, zeigt sich darin, daß beide die Tendenz besitzen, in das orangefarbige Mischsalz überzugehen. Auch in der wässerigen Lösung ist die Isomerie vorhanden; ein wichtiger Beweis dafür, daß die Verschiedenheit der Salze nicht als ,physikalische Isomerie“ gedeutet werden kann. Die frisch bereiteten wässerigen Lösungen des gelben und roten Salzes zeigen ähnliche Farbunterschiede wie die festen Salze und streben, wie zu erwarten, dem Zustande des Mischsalzes zu : die Lösungen des rein gelben Salzes werden schon nach Verlauf einiger Stunden orange, während die Farbaufhellung der Lösungen des roton Salzes mehrere Wochen in Anspruch nimmt.

Die Resultate der Salzbildung bei diesem $\mathrm{m}$ Nitrophenol sind insofern noch von allgemeinerem

44) Hantzsch, Berl. Berichte 40, 1523 . 
Interesse, als dadurch im Prinzip die Existenz von $\mathrm{m}$-Chinoiden bewiesen ist.

Ähnliche Erscheinungen treten bei der

b) Salzbildung bei Nitroketonen ${ }^{44}$ )

mit der Gruppe CO.CH( $\left.\mathrm{NO}_{2}\right)$ - auf. Durch Enolisierung geht letztere in die Grupe $\mathrm{C}(\mathrm{OH}) \mathrm{C}\left(\mathrm{NO}_{2}\right)$ über, die auch in den o-Nitrophenolen vorhanden ist. Tatsächlich sind auch die Verhältnisse der Salzbildung vielfach analoge mit dem Unterschiede, daB in einigen Fällen neben gelben und roten Salzen (chromo-Salzen) noch farblose (leuko-Salze) auftreten.

Die Existenz der farblosen Salze beweist direkt, daß die Gruppe - OMe im Gegensatz zu K a u f $\mathbf{f}$ ma $n \mathrm{n}$ kein Auxochrom sein kann. Der Ubergang der leuko- in die chromo-Salze läßt sich vielleicht durch folgendes Schema darstellen :

$$
\begin{aligned}
& \text { C: }: 0 \\
& \mathrm{C}-\mathrm{O} \\
& \text { C: NO.OMe } \\
& \text { C-No.OMe } \\
& \text { leuko-Salze, } \\
& \text { chromo-silze. }
\end{aligned}
$$

Die spezielle Konstitution der gelben und roten Salze lkann wie bei den Nitrophenolen, noch nicht mit Sicherheit angegeben werden. Die Salze aus Nitromalonamid, Nitromalonester und Nitrobarbitursäure sind in festem Zustande farblos, doch bilden letztere beiden schon gelbe Ionen. Dimethylnitrobarbitursäure gibt schon überwiegend gelbe Salze, nur die $\mathrm{NH}_{4}, \mathrm{Ag}$ und $\mathrm{Hg}$-Salze sind noch farblos. Beim Phenylmethylnitropyrazolon sind nur noch die $\mathrm{Ag}$ - und $\mathrm{Hg}$-Salze farblos, neben den gelben treten aber hier bereits die isomeren roten Alkalisalze auf.

Weit komplizierter gestalten sich die Verhältnisse bei der Salzbildung der Dinitroverbindungen ${ }^{45}$ ) z. B. bei Nitrophenylnitromethan

$$
\mathrm{NO}_{2} \backslash>\mathrm{CH}_{2} \mathrm{NO}_{2}
$$

wo auBer gelben und roten noch grüne und violette Salze aufgefunden sind. Auch hier kann man die Existenz dieser Isomeren mit Hilfe der Umlagerungstheorie einigermaßen plausibel machen.

c) Salzbildung bei Phenolaldehyden, Phenolketonen, Phenolearbonsäuren und Derivaten.

Den Nitrophenolen sind inbezug auf die Salzbildung auch die Aldehydphenole zu vergleichen ${ }^{46}$ ). Die Alkyl- and Acylderivate dieser Verbindungen von eindeutiger Konstitution, z. B. $\mathrm{C}_{6} \mathrm{H}_{4} \cdot \mathrm{OCH}_{3} . \mathrm{CHO}$ sind ohne Ausnahme farblos; wie die entsprechenden Nitrophenolkörper. Die freien Wasserstoffverbindungen sind auch meist farblos, in der o-Reihe tritt aber durch Einführung gewisser Radikale Farbe auf, wie bei (4) $\mathrm{RO} . \mathrm{C}_{6} \mathrm{H}_{3} . \mathrm{OH} . \mathrm{CHO}$, die in festem $\mathrm{Zu}$ stande gelb und dem o-Nitrophenol vergleichbar, als teilweise isomerisierte chromo-Aldehydphenole, a. h. als Gemische resp. feste Lösungen der beiden Formen :

45) H antzs ch, Eerl. Berichte 40, 1533.

46) Hantzs ch, Berl. Berichte 39, 3092.

$$
\begin{aligned}
& 120 \cdot \mathrm{C}_{6} \mathrm{H}_{3}^{\mathrm{OH} \cdot \mathrm{OH}} \\
& \text { gelb } \\
& \text { und } \mathrm{RO} \cdot \mathrm{C}_{6} \mathrm{H}_{3} \mathrm{CHO}_{\text {farblos }}^{\mathrm{OH}}
\end{aligned}
$$

aufzufassen sind.

Die Salze der Oxyaldehyde leiten sich sowohl von den echten als auch isomerisierten chromo. Aldehydphenolen $a b$ und sind dementsprechend sowohl farblos als gelb. Sämtliche Salze. und Ionen aus Paraoxybenzaldehyd sowie seinem MonobromundDibromderivate sind farblos; dio Salze aus m-Oxy benzaldehyd sind in festem und wasserfreiem $\mathrm{Zu}$ stande ebenfalls sämtlich farblos, geben aber gelbe wässerige Lösungen. Erst beim o-Oxybenzaldehyd, dem wie dem o-Nitrophenol die größte Neigung zu Isomerisation inne wohnt, treten neben farblosen (z. B. $\mathrm{NH}_{4^{-}}$) auch gelbe (z. B. K-) Salze auf.

Ahnliche Verhältnisse liegen bei der Isomerisation von aromatischen Oxyketonen, Oxychromonen und Oxyxanthonen, sowie den Oxybenzoesäurederivaten vor, bei letzteren sind die beim Hydrochinondicarbonsäureester erhaltenen Resultate am interessantesten und befriedigend im Sinne der Umlagerungstheorie zu deuten ${ }^{47}$ ).

$$
\begin{array}{ccc}
\mathrm{COOC}_{2} \mathrm{H}_{5} \\
\mathrm{HO} \cdot \mathrm{COH} \\
\mathrm{COOC}
\end{array}
$$

d) Um lagerungen bei der Salzbildung der Tripheny Imethanfarbstoffe.

Von größtem Interesse für die Farbstoffohemiker sind die unzählige Male studierten Farberscheinungen bei den Triphenylmethanfarbstoffen, wo man bekanntlich nach dem Vorgange $\mathrm{Nietzk}$ is zuerst das Auftreten von Farbe mit der Bildung chinoider Formen in Beziehung brachte.

Die von Rosenstieh I für die Farbstoffe der Aminotriphenylmethanreihe vorgeschlagene Formel, die in etwas modifizierter Form kürzlich von Baeyer wieder zur Diskussion gestellt wurde, kann mit Rüicksicht auf die im vorletzten Kapitel zu besprechenden Arbeiten G o mbergs wohl als widerlegt betrachtet werden.

$$
\text { Fuchsin: }
$$

$$
\begin{array}{cc}
\text { Chinoidformel } & \text { 11ach Rosenstiehl } \\
\left(\mathrm{NH}_{2} \mathrm{C}_{6} \mathrm{H}_{4}\right\rangle_{2} \mathrm{C}: \mathrm{C}_{6} \mathrm{H}_{4}: \mathrm{NH}_{2} \mathrm{Cl} & \left(\mathrm{NH}_{2} \mathrm{C}_{6} \mathrm{H}_{4}\right)_{3} \mathrm{C} \cdot \mathrm{Cl}
\end{array} \text {. }
$$

$\mathrm{Um}$ noch ein weiteres Beispiel zu nennen, sei an die Bildung der roten Alkalisalze aus de $\mathrm{m}$ fa $\mathrm{r} b$ losen Phenolphthalein erinnert, die, worauf u. a. S t i e g lit $\mathrm{z}^{48}$ ) mit Nachdruck verwies, im Sinne folgender Umlagerung zu deuten ist:

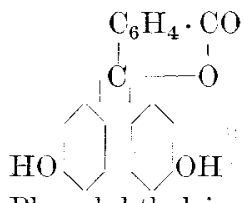

Phenolphthalein, Lacton, farblos,

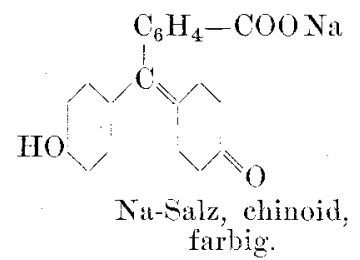

47) H a n t z s h, Berl. Berichte 39, 1392

$\left.{ }^{4}\right)$ J. Am. Chem. Soc. 25, 1112. 
Fine schöne Bestätigung dieser Ansichten brachte kürzlich eine Arbeit von R. M e yer und Marx $\left.{ }^{49}\right)$, die nach der Methode von Hantzsch und G or k e durch Alkylierung des Silbersalzes des Tetrabromphenolphthaleins einen chinoiden, intensiv gelben Diäthylester von der Konstitution I erhielten, der wie die chromo-Nitrophenoläther labil ist und sich in den lactoiden, farblosen Äther IT

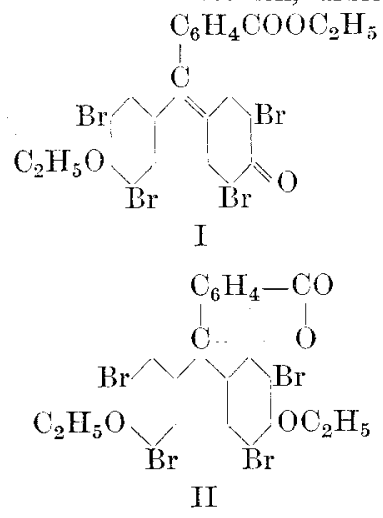

umwandelt. Daraus ist wohl mit ziemlicher Sicherheit zu schließen, daß auch den Salzen des Phenolphthaleins die dem Äther I analoge, d. h. chinoide Struktur zukommt.

Die Bildung der Triphenylmethanfarbstoffsalze ist schon früher eingehend von $\mathrm{H}$ a $\mathrm{t} \mathrm{zs} \mathrm{ch}$ und $\mathrm{Os}$ s w a l $\mathrm{d}^{50}$ ) im Anschlu $B$ an die Untersuchungen überPse $\mathbf{P}$ doba se $n$ studiert worden. Andiese Untersuchungen soll mit Rücksicht auf die im nächsten Kap. zu besprechenden Arbeiten Gom bergs über die Konstitution der Triphenylmethanfarbstoffe erinnert werden. Pseudobasen sind bekanntlich elektrisch neutrale Hydroxylverbindungen, die aus den echten mit ihnen isomeren Ammoniumbasen durch eine intramolekulare Umlagerung hervorgehen und zwar meist dadurch, daß das Hydroxyl rom Stickstoff zum Kohlenstoff wandert. Eine gleiche Umlagerung erleiden die Pseudobasen bei der Salzbildung; wie die Pseudosäuren sind sie hierzu nicht direkt befähigt, sondern nur unter gleichzeitiger intramolekularer Umstellung. Eines der einfachsten Beispiele dieser Art bieten die Acridiniumverbindungen: aus den quaternären Salzen, z. B. dem Chlorid des Phenylmethylacridiniums I

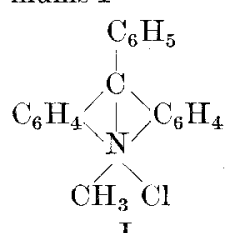

I

Salz der echten Base

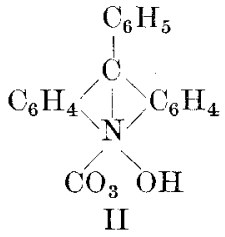

echte Base

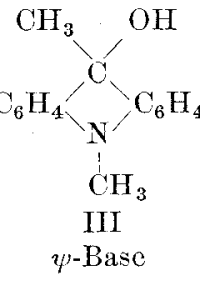

wird durch Kali primär die echte Ammoniumbase II in Freiheit gesetzt, die von der Stärke des Kalis ist. Thre Existenz ist aber nur vorübergehend, sie hat die Tendenz, sich in die isomere Pseudobase (Phenylmethylacridol) umzulagern, die ein elektrisch neutrales Derivat des dreiwertigen Stickstoffs darstellt.

49) Berl. Berichte 40, 1414.

50) Berl. Berichte 33, 278.
Ahnliche Umwandlungen erleiden viele den Farbstoffsalzen entsprechende Farbbasen, wofür der Beweis in der umfassenden Arbeit von $\mathrm{H}$ an $\mathrm{tzsch}$ und $\mathrm{O}$ s s w a $1 \mathrm{~d}$ erbracht ist. Das Farbstoffsalz (Krystallviolett, Pararosanilin, Brillantgrün) ist nach dieser Ansicht, die sich mit der von $\mathrm{N}$ i e t $\mathrm{z} \mathrm{k}$ i u. a. vertrotenen deckt, das Salz einer echten quaternären Ammoniumbase von chinoidem Typus, aus dem durch Basen primär die chte Farbbase vom Charakter der vollständig substituierten Ammoniumbasen in Freiheit gesetzt wird, die sich aber in der Lösung mehr oder weniger rasch isomerisiert, indem das ursprünglich als Ion vorhandene Hydroxyl sich an dem in p-Stellung befindlichen Methankohlenstoffatom festsetzt, wobei gleichzeitig die chinoide Gruppierung in die benzoide übergeht. Das allgemeine Schema einer derartigen Umwandlung ist folgendes:

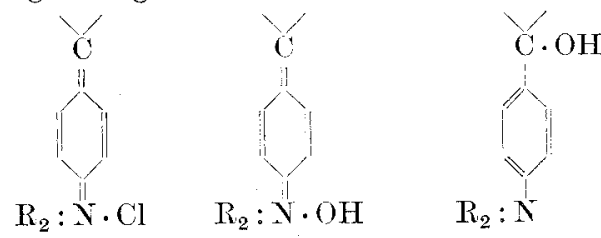

Farbsalz, echte Ammonium- farblose $\psi$-Anfarbibase, labil, moniumbase

Carbinol, stabil.

Beim Krystallviolett, wo sich die Umwandlung am besten beobachten ließ, ist die Umlagerung der Farbbase in die $\psi$-Base folgendermaßen $\mathrm{zu}$ formulieren :

$$
\begin{aligned}
& {\left[\left(\mathrm{CH}_{3}\right)_{2} \mathrm{NC}_{6} \mathrm{H}_{4}\right]_{2} \mathrm{C}=\mathrm{N}\left(\mathrm{CH}_{3}\right)_{2} \mathrm{OH}} \\
& {\left[\left(\mathrm{CH}_{3}\right)_{2} \mathrm{NC}_{6} \mathrm{H}_{4}\right]_{2} \mathrm{C}(\mathrm{OH})=\mathrm{N}\left(\mathrm{CH}_{3}\right)_{2} .}
\end{aligned}
$$

Den bei den Triphenylmethanfarbstoffen beobachteten Umlagerungen in gewisser Weise verwandt sind die besonders von $W$ erner ${ }^{51}$ ) studierten Bildungen

e) farbigersalze bei Carboxonium und Carbothioniumverbindungen, die mit den basischen Eigenschaften des Sauer. stoffs im Zusammenhang stehen. Bekanntlich liegen in den salzartigen Verbindungen des Dimethylpyrons nach $\mathrm{Col}$ I i e und $\mathrm{Ti}$ e $\mathrm{k}$ le, sowie nach $\mathrm{B}$ a e y e r Oxoniumverbindungen vor, die als Analoge der Ammoniumverbindungen aufzufassen sind. Wie in

$$
\begin{array}{ccc}
\mathrm{CO} \\
\mathrm{CH}_{3} \cdot \mathrm{C} & \mathrm{CH} \cdot \mathrm{CH}_{3} \\
\mathrm{H} & \mathrm{Ol}
\end{array}
$$

diesen der Stickstoff meist als fünfwertig angenom men wird, so werden die Oxoniumverbindungen gewöhnlich mit sog. vierwertigem Sauerstoff formuliert. Richtiger ist es aber, nach W ern e r s ${ }^{52}$ ) Vorgange die Oxoniumsalze analog den Ammoniumsalzen als einfachste Komplexsalze anzusehen, bei

51) Berl. Ber chte 34, 3300; vg]. K e h r m a n n 32,$2601 ; 34,1623$

52) Liebigs Ann. 322, 261; s. a. We rner, Neuere Anschauungen auf dem Gebiete der anorganischen Chemic, Vieweg, Braunschweig, 1907. 
denen die Ammoniak- resp. Sauerstoffverbindungen vom Wasserstoffatom der Säure HX durch sogen. Nebenvalenzen gebunden werden.

$\mathrm{NH}_{4} \mathrm{X}$ Ammoniumsalze: $\left(\mathrm{NH}_{3} \ldots \mathrm{H}\right) \mathrm{X}$ $\mathrm{NH}_{3} \mathrm{C}_{6} \mathrm{H}_{5} \mathrm{X}$ Aniliniumsalze : $\left(\mathrm{NH}_{2} \mathrm{C}_{6} \mathrm{H}_{3} \ldots \mathrm{H}\right) \mathrm{X}$

$$
\begin{gathered}
\mathrm{CO}, \mathrm{CH}-\mathrm{CR} \text { OHX Pyroniumsalz: } \\
(\mathrm{CH}-\mathrm{CR}-\mathrm{CH}-\mathrm{CR} \\
\mathrm{CH}-\mathrm{CR}
\end{gathered}
$$

Als Beispiel farbiger Carboxoniumverbindungen seien die Salze des Xanthydrols :

$$
\mathrm{C}_{6} \mathrm{H}_{4} \mathrm{O}_{6} \mathrm{H}_{4}
$$

genannt; letztere Verbindung ist als Pseudoxanthoniumbase I aufzufassen, die den früher besprochenen Pseudobasen der stickstoffhaltigen Triphenylmethanfarbstoffe an die Seite zu stellen ist, und die sich in Mineralsäuren mit intensiv gelber Farbe zu einem Xanthoniumsalze II löst, das somit von der in freiem Zustande nicht existenzfähigen Xanthoniumbase abzuleiten ist. Die Xanthoniumsalze konnten in Form beständiger Doppelsalze isoliert

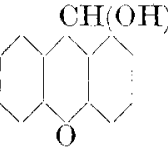

I $y$-Base

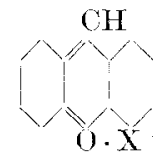

II Salz d.echten Base

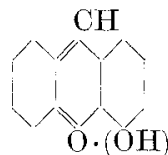

III echte Base werden. Ahnlich ist die Bildung farbiger Salze beim Pheno-a-naphtoxanthydrol IV sowie beim Thioxanthydrol V zu erklären:
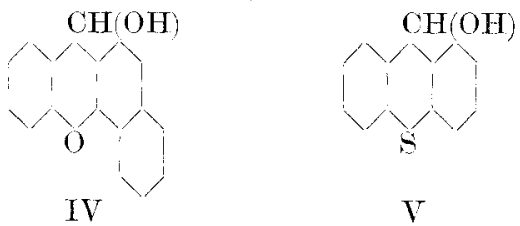

Das Auftreten von Farbe bei diesen Oxoniumresp. Thioniumverbindungen ist wohl so $\mathrm{zu}$ deuten, daß gleichzeitig mit der Bildung des Oxoniumsalzes eine orthochinoide Atomgruppierung geschaffen wird.

$\mathrm{K}$ e $\mathrm{h}$ r $\mathrm{m}$ a $\mathrm{n} \mathrm{n}$ s Ansicht, daß in gewissen $\mathrm{Ox}$ azin- und Thiazinfarbstoffen ein durch vierwertigen Sauerstoff bedingter o-Chinonchromophor enthalten sein soll im Sinne der Formeln :
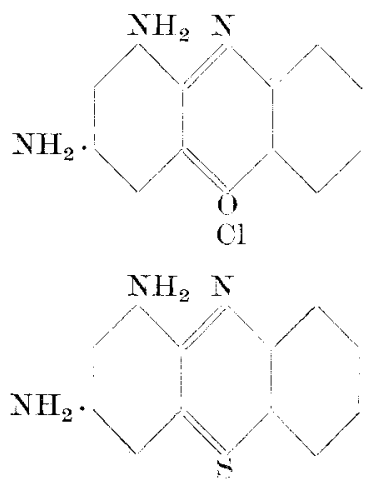

(i) ist nach Untersuchungen von $\mathrm{Han} \mathrm{tzs} c h$ nicht wahrscheinlich, vielmehr dürften in diesen Verbindungen, sowie im sogen. L a $u t h$ schen Violett, $\mathrm{p}$ Chinoide vorliegen, in denen der Sauertsoff normaler Weise zweiwertig ist:
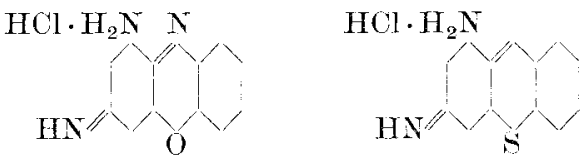

f) Farbige Salzedes Triphenylcar. binols. Chinocarboniumsalze.

Die zuerst von $\mathrm{N}$ ie t zki vertretene Ansicht, da $B$ die Farbigkeit der Triphenylmethanfarbstoffe auf einer chinoiden Atomgruppierung beruht, erhält eine äußerst wichtige Stütze durch die neuesten Arbeiten von $\mathrm{G}$ o $\mathrm{m}$ b e r g über farbige Salze, die sich vom Triphenylcarbinol ableiten, und die im Anschluß an dio ausgezeichneten Arbeiten über das Triphenylmethyl entstanden sind.

Bekanntlich bilden die an sich farblosen Chloride und Bromide $\left(\mathrm{C}_{6} \mathrm{H}_{5}\right)_{3} \mathrm{ClCl}$ und $\left(\mathrm{C}_{6} \mathrm{H}_{5}\right)_{3} \mathrm{CBr}$ mit flüssigem Schwefeldioxyd intensiv gelbe Lösungen, die die Elektrizität leiten ${ }^{5}{ }^{3}$, Triphenvlcarbinole $\left(\mathrm{C}_{6} \mathrm{H}_{5}\right)_{3} \mathrm{C}(\mathrm{OH})$ sowie dessen Substitutionsprodukte lösen sich in konz. Schwefelsäure mit tiefroter Farbe, das von $\mathrm{G}$ o $\mathrm{mb}$ e $\mathrm{r} g$ und $\mathrm{Con} \mathrm{e}^{\overline{4}}$ ) zuerst dargestellte normale Sulfat $\left[\left(\mathrm{C}_{6} \mathrm{H}_{5}\right)_{3} \mathrm{C}\right]_{2} \mathrm{SO}_{4}$ ist auch in festem Zustande rot. Von großem Interesse für die Frage sind ferner die von $B$ a e y e r ${ }^{55}$ ) eingehend untersuchten sauren Sulfate:

(Cl. $\left.\mathrm{C}_{6} \mathrm{H}_{4}\right)_{3} \mathrm{C} . \mathrm{SO}_{4} \mathrm{H} . \mathrm{H}_{2} \mathrm{SO}_{4}$ : braun, $\left(\text { J. } \mathrm{C}_{6} \mathrm{H}_{4}\right)_{3} \mathrm{C} . \mathrm{SO}_{4} \mathrm{H} . \mathrm{H}_{2} \mathrm{SO}_{4}$ : braun, mit grünem Metallglanz, sowie die Zinnchloridadditionsproduk to $\left(\mathrm{C}_{6} \mathrm{H}_{5}\right)_{3} \mathrm{C} . \mathrm{Cl} . \mathrm{SnCl}_{4}$ : gelb $\left(\mathrm{ClC}_{6} \mathrm{H}_{4}\right)_{3} \mathrm{C} . \mathrm{Cl} . \mathrm{SnCl}_{4}$ : rot.

Auch das von $G$ om be r g ${ }^{56}$ ) isolierte Triphenylmethyl löst sich in einigen Solvenzien intensiv farbig. Für die von $\mathrm{N}$ or r is und $\mathrm{S}$ a nders ent deckten, von $\mathrm{Kehrman} \mathrm{n}^{57}$ ) eingehender studierten farbigen, salzartigen Verbindungen des Triphenylcarbinols hielt letzterer eine chinoide (oder besser chinoloide) Struktur wahrscheinlich, z. B.

$$
\underset{\mathrm{CH}_{3} \mathrm{OC}_{6} \mathrm{H}_{4}}{\mathrm{CH}_{3} \mathrm{OC}_{6} \mathrm{H}_{4}} \mathrm{C}_{\text {Trianisylearbinols, }}^{\mathrm{OCH}_{3}}
$$

während $B$ a e y e r in neweren Arbeiten ${ }^{58}$ ) eine chinoide Gruppierung in Abrede stellt. Vielmehr macht $B$ a e y e r die Annahme, da 3 die salzartigen Verbindungen des Triphenylcarbinols in zwei verschiedenen Z11ständen existieren können, einem farblosen, nicht ionisierten und einem farbigen ionisierten $\left[\mathrm{z} . \mathrm{B} .\left(\mathrm{C}_{6} \mathrm{H}_{5}\right)_{3} \mathrm{CCl}\right.$ in flüss. $\left.\mathrm{SO}_{2}\right]$; der Übergang des einen Zustandes in den anderen soll mit einer bis jetzt nicht genau zu definierenden Veränderung in der Natur des Triphenylmethyls verbun-

$\left.{ }^{53}\right) \mathrm{W}$ a l d e n, Z. physikal. Chem. 43, 385; Berl. Berichte 3\%, 2018; vgl. G o m ber $\mathrm{g}$, Berl. Berichte 35, 2397.
54) Berl. Berichte $3 \%$, 3543.
55) Berl. Berichte 38, 569, 1156
56) Berl. Berichte 33, 3150
57 ) Berl. Berichte 34, 3815 .
58) Berl. Berichte 38, 569 . 
den sein. Das neue Moment in der $\mathrm{B}$ a e y e $\mathrm{r}$ schen Spekulation ist somit die Vorstellung, daß bei gewissen Carboniumverbindungen durch Betätigung gewisser Valenzen, Carboniumvalenzen, die auch durch eine besondere Formulierung : $\left(\mathrm{C}_{6} \mathrm{H}_{5}\right)_{3} \mathrm{C} \cdots$ $\mathrm{Cl},\left(\mathrm{C}_{6} \mathrm{H}_{5}\right)_{3} \mathrm{C} \sim \mathrm{SO}_{4} \mathrm{H}$ kenntlich gemacht werden, Dissoziation und gleichzeitig Farbigkeit hervorgerufen werde. Wesentlich für diese Auffassung ist, daß der z. B. beim Lösen des Carbinols in konz. Schwefelsäure sich zwischen dem Rest $\left(\mathrm{RC}_{6} \mathrm{H}_{4}\right)_{3} \mathrm{C}$ und dem Radikal $\mathrm{x}$ abspielende Dissoziationsvorgang die Farbe bedingt, daß aber die Radikale $\left(\mathrm{RC}_{6} \mathrm{H}_{4}\right)$ keine Änderung ihrer Funktionen erleiden.

Es hat somit den Anschein, als ob sichdie genannten Salze von einer Base, Carboniumbase, dem Triphenylcarbinol $\left(\mathrm{C}_{6} \mathrm{H}_{5}\right)_{3} \mathrm{COH}$ ableiten, in der das Triphenylmethyl die Rolle eines schwach elektropositiven Metalles spielt. Von weiterer Bedeutung fïr die Frage sind ferner B a e y er s Untersuchungen ïber die methoxylierten Triphenylcarbinole. Durch Einführung der Methoxylgruppe erhöht sich nämlich die Basizität des Triphenylcarbinols, und zwar ist der Einfluß am stärksten in der p-, am schwächsten in der m-Stellung, was sich überzeugend dadurch nachweisen ließ, daß die Lösung des m-substituierten Carbinols in Eisessigschwefelsäure, die das farbige Sulfat enthalten, durch geringere Mengen von wässerigem Alkohol entfärbt, d. h. hydrolysiert wird, als die Lösung der $p$-Verbindung. Bemerkenswert ist ferner, daß die Basizität steigt mit der Zahl der eingeführten Methoxyle und zwar im Verhältnis der Potenzen dieser Zahlen, wobei wieder die genannte Alkoholmenge als Taß für die Basizität angesehen wird. Auch für die methoxylsubstituierten Derivate des Dibenzalacetons $\mathrm{C}_{6} \mathrm{H}_{5} \mathrm{CH}: \mathrm{CH}_{2} \mathrm{COCH}: \mathrm{CHC}_{6} \mathrm{H}_{5}$, die ebenfalls farbige Sulfate bilden, ist das ,Potenzengesetz ${ }^{6}$ gültig. B a e y e r nennt die Erscheinung, daß farblose oder schwach farbige Stoffe mit Säuren farbige, salzartige Verbindungen liefern, ohne daß Umlagerung in eine chinoide Gruppierung vor sich geht, Halochromie.

In einer neuen Abhandlung weist nun $G$ o m berge0) im Gegensatz zu B a eyer nach, daß mit der Bildung der farbigen Derivate des Triphenylcarbinols doch eine Umlagerung stattfindet, woraus weiter der Schluß zu ziehen ist, daß sämtliche farbigen Derivate des Triphenylmethans chinoid konstituiert sind.

Diese Erkenntnis leitete Gom berg aus folgenden Versuchen ab. Triphenylmethylchlorid sowie dessen Homologe reagieren mit Silbersulfat bei Anwendung geeigneter Lösungsmittel glatt unter Abspaltung des Carbinolchlors, wobei die Intensiv farbigen Sulfate entstehen. Ahnlich verhalten sich die halogenierten Triphenyl-

59) Berl.Berichte 35, 3021.

6v) Berl. Berichte 40. 1847. Nach einer wäbrend der Korrektur erschicnenen Arbeit v. Ba eyers (Berl. Ber. 40, 3083) sind jedoch dio Versuche Go m bergs für die Frage der chinoiden Struktur der Salze des Triphenyicarbinols nicht beweiskräftig, sodaß die vielumstrittene Frage nach der Konstitution dem Salze vorläufig als ungelöst betrachtet werden muB. Die Arbeit v, $\mathrm{B}$ a e yers konnte leider nicht mehr berücksichtigt werden. methylchloride, z. B. Tri-p-bromtriphenylmethylchlorid $\left(\mathrm{BrC}_{6} \mathrm{H}_{4}\right)_{3} \mathrm{CCl}$ gegen Silbersulfat. Auch in diesem Falle treten intensive Färbungen auf. Wären diese im Sinne B a e y e $r$ s auf Bildung eines halochromen Carbinolsulfates I zurückzuführen, so sollte nur das

$$
\begin{aligned}
& \text { I. }\left(\mathrm{BrC}_{6} \mathrm{H}_{4}\right)_{3} \mathrm{C} \cdot \mathrm{SO}_{4} \cdot \underset{\left.\mathrm{C}_{6} \mathrm{H}_{4} \mathrm{Br}\right)_{3}}{\mathrm{Br} \mathrm{Br}} \\
& \text { II. }\left(\mathrm{BrC}_{6} \mathrm{H}_{4}\right)_{2} \mathrm{C}: \mathrm{C}_{6} \mathrm{H}_{4}-\mathrm{SO}_{4}-\mathrm{C}_{6} \mathrm{H}_{4}:\left(\mathrm{C}_{6} \mathrm{H}_{4} \mathrm{Br}\right)_{2}
\end{aligned}
$$
III. $\left(\mathrm{BrC}_{6} \mathrm{H}_{4}\right)_{2} \mathrm{C}: \mathrm{C}_{6} \mathrm{H}_{4}: \mathrm{SO}_{4}$.

Carbinolhalogen herausgenommen werden; verdankt hingegen die Farbe einer chinoiden (oder chinoloiden) Gruppierung ihre Entstehung, so müßte, worauf schon $\mathrm{B}$ a e y er hingewiesen hatte, das Halogen in dem chinoiden Ring labil werden, und bei UberschuB von Silbersulfat sollte auch das eine Ringhalogen abgespalten werden, was die Entstehung einer Verbindung im Gefolge haben würde, die etwa gemäß Formel III gebaut wäre. In der Tat entschied der Versuch im letzten Sinne; so wird bei der tribromierten Verbindung unter geeigneten Versuchsbedingungen außer dem Carbinolchlor genau 1 Atom Ringhalogen herausgenommen, was nur unter Umstellung in eine chinoloide Form möglich ist. In ähnlicher Weise konnte auch bei den Carbinolchloriden Tautomerisation exakt nachgewiesen werden. Die in festem Zustande wie in indifferenten Medien Benzol, Ather, absolut farblosen Carbinolchloride lösen sich in flüssigem Schwefeldioxyd mit gelber bis roter Farbe und können aus diesen Lösungen nach dem Verdunsten des Mediums in der ursprünglich farblosen Form zürückerhalten werden. Besteht nun zwischen Farbe und Konstitution der bei den Sulfaten nachgewiesene Zusammenhang, so sollte man auch ein verschiedenes Verhalten der Carbinolchloride in den farblosen Lösungen (Benzol, Äther) einerseits und den farbigen Lösungen $\left(\mathrm{SO}_{2}\right)$ andererseits erwarten. Die parabromierten Triphenylmethylchloride verhalten sich nun tatsächlich in den genannten Solvenzien gegenüber trockenem Chlorsilber verschieden; in rein benzolischer Lösung ist letzteres ohne Einwirkung auf das Chlorid, während bei Gegenwart von flüssigem Schwefeldioxyd und Dimethylsulfat als Lösungsmittel das Ringbrom durch Chlor ersetzt wird, und zwar werden nacheinander sämtliche drei Bromatome gegen Chlor ausgetauscht. Diese durch ein so milde wirkendes Reagens wie Chlorsilber, bei einer derartig stabilen Verbindung wie Tribromtriphenylmethylchlorid bewirkte Reaktion läßt sich nur so verstehen, daß das flüssige Schwefeldioxyd auf die Triphenylmethanverbindung eine tautomerisierende Wirkung ausuibt. Go m b e r g macht die plausible Annahme, daß sich zunächst ein Gleichgewicht zwischen den beiden folgenden Formon herherstellt :

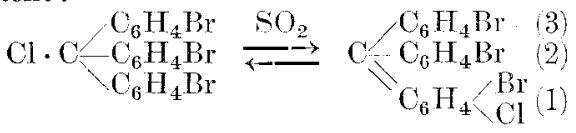

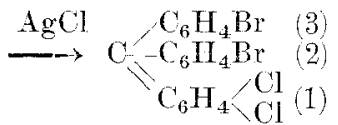

und gleichzeitig das Brom in dem chinoid gewordenen Ringe (1) durch Chlor ersetzt wird. Die so ge- 
bildete chinoide Form des Chlordibromtriphenylmethylchlorids wird sich mit einer gewissen Menge der benzoiden Form ins Gleichgewicht setzen :

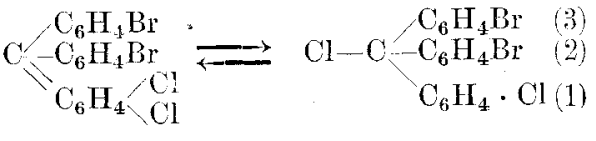

$$
\begin{aligned}
& \stackrel{\mathrm{SO}_{2}}{\longrightarrow} \mathrm{C}_{6} \mathrm{H}_{4} \mathrm{Br}(3) \\
& \mathrm{C}_{6} \mathrm{H}_{4} \cdot \mathrm{Cl}(1)
\end{aligned}
$$

und letztere unter dem Einfluß des Dioxyds eine weitere Tautomerisation erleiden, indem jetzt der Ring (2) chinoid wird, und das darin enthaltene bewegliche Brom der Wirkung des Chlorsilbers anheimfällt, wodureh die chinoide Form des Monobromdichlortriphenylchlorids entsteht. Man kann sich so vorstellen, daß bei genügend langer Einwirkung des Chlorsilbers in sämtlichen drei Ringen das Brom durch Chlor ersetzt wird, so daß schließlich als Endprodukt nach Abdunsten des Lösungsmittels Trichlortriphenylmethylchlorid erscheint.

Diese Tatsachen zwingen nach Gomberg nun zu der Annahme, daß in den farbigen Chloriden und Sulfaten des Triphenylcarbinols $\mathrm{S}$ a $\mathrm{l}$ z e vorliegen, die sich von einer $\mathrm{C} h$ inoc a $\mathbf{r}$ bon i u mase:

$$
\mathrm{R}_{2} \mathrm{C}:=\mathrm{C}_{\mathrm{OH}}^{\mathrm{X}}
$$

d. i. einem sekundären Chinol ableiten. In flüssigem Schwefeldioxyd ist diese Base sowie deren Salze nach W a $1 \mathrm{~d}$ e $\mathrm{n}$ s Messungen als dissoziiert anzusehen. Von den durch Tautomerisation entstehenden chinoloiden Formen leiten sich ferner die farbigen Doppelsalze der Carbinolchloride mit gewissen Metallhalogeniden ab, deren Formel somit durch folgendes Symbol dargestellt werden müßte:

$$
\mathrm{R}_{2} \mathrm{C}:=\mathrm{C}=\begin{aligned}
& \mathrm{X} \quad \mathrm{X}=\mathrm{Cl}, \mathrm{Br} \\
& \mathrm{Cl}, / \mathrm{nCl}_{2}, \mathrm{SnCl}_{4}, \mathrm{SbCl}_{3}
\end{aligned} .
$$

Ferner folgt mit Notwendigkeit, daß auch die zahlreichen Diphenylmethanderivate, z. B. Benzo. phenonchlorid $\left.{ }^{61}\right): \mathrm{C}_{6} \mathrm{H}_{5} \mathrm{CCl}_{2} \mathrm{C}_{6} \mathrm{H}_{5}$, Dicinnamenyldichlormethan $\left.{ }^{62}\right)\left(\mathrm{C}_{6} \mathrm{H}_{5} \mathrm{CH}: \mathrm{CH}\right)_{2} \mathrm{CCl}_{2}$, das vielfach untersuchte Dibenzalaceton $\mathrm{C}_{6} \mathrm{H}_{5} \mathrm{CH}: \mathrm{CH} . \mathrm{CO}: \mathrm{CH}$. : $\mathrm{CHC}_{6} \mathrm{H}_{5}$, die sämtlich farbige Sulfate resp. Doppelsalze liefern, ähnlich wie die Derivate des Triphenylcarbinols in zwei Zuständen, einem benzoiden, farblosen und einem chinoiden, farbigen existieren müssen. Unter diesen Gesichtspunkten ist auch das Verhalten der interessanten von $\mathrm{Sehm}$ id $\left(\mathbf{i n}{ }^{82 \mathrm{a}}\right.$ ) entdeckten Magnesiumverbindung des Triphenylchlormethans verständlich, die ebenfalls in zwei Formen einer chinoiden und benzoiden besteht.

Auch für die Frage nach der Konstitution des ebenfalls in zwei Zuständen existierenden Triphenylmethyls selbst hat die Untersuchung wichtige Anhaltspunkte geliefert. Möglicherweise ist das feste, farblose Triphenylmethyl identisch mit Hexaphenyläthans in den farbigen Löungen der Verbindung wäre damn folgendes Gleichgewicht zwischen der benzoiden und chinoiden Form anzunehmen:

61) Berl. Berichte 35, 2405, 1887.

62) Straus und Ecker, Berl. Berichte 39, 2977 ,

(2a) Berl. Ber. 39. 628, 4183.

$$
\begin{aligned}
& \mathrm{O}_{6} \mathrm{H}_{5} \\
& \mathrm{C}_{6} \mathrm{H}_{5} \\
& \mathrm{C}_{6} \mathrm{H}_{5} \\
& \mathrm{C}_{\left(\mathrm{C}_{6} \mathrm{H}_{5}\right)_{3}}
\end{aligned} \mathrm{C}^{\mathrm{C}_{6} \mathrm{H}_{5}} \begin{aligned}
& \mathrm{O}_{6} \mathrm{H}_{5} \\
& \mathrm{C}_{6} \mathrm{H}_{4}
\end{aligned} \mathrm{C}_{\mathrm{C}\left(\mathrm{C}_{6} \mathrm{H}_{5}\right)_{3}}^{\mathrm{H}}
$$

Die beträchtliche Leitfähigkeit, die dieser merkwürdige Kohlenwasserstoff in flüssigem Schwefeldioxyd aufweist ${ }^{63}$ ), würde durch die Annahme einer elektrolytischen Dissoziation zufolge des Schemas:

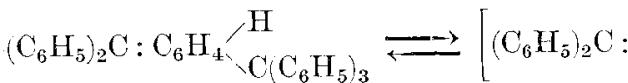

$$
\begin{aligned}
& \left.\left.\mathrm{C}_{6} \mathrm{H}_{4}\right]^{\mathrm{H}}\right]^{+}+\left[\left(\mathrm{C}_{6} \mathrm{H}_{5}\right)_{3} \mathrm{C}\right]
\end{aligned}
$$

verständlich.

Schließlich lassen sich mit Hilfe der Annahme intermediärer Bildung der Chinocarboniumverbindungen auch die Entstehung der eigentlichen Triphenylmethanfarbstoffsalze aus den Carbinolen plausibel machen. Die Bildung des Parafuchsins aus dem Pararosanilin unter dem Einfluß von Säuren. denkt sich G o m b e r g in folgender Weise :

$$
\begin{aligned}
& \left(\mathrm{NH}_{2} \mathrm{C}_{6} \mathrm{H}_{4}\right)_{2} \mathrm{C}-\mathrm{C}_{6} \mathrm{H}_{4} \mathrm{NH}_{2} \\
& \mathrm{OH} \\
& \text { Pararosanilin } \\
& \begin{array}{c}
\left.\rightarrow \mathrm{NH}_{2} \mathrm{O}_{6} \mathrm{H}_{4}\right)_{2} \mathrm{C}-\mathrm{C}_{6} \mathrm{H}_{4} / \mathrm{OHI} \\
\text { Chinol }
\end{array} \\
& \rightarrow\left(\mathrm{NH}_{2} \mathrm{C}_{6} \mathrm{H}_{4}\right)_{4} \mathrm{C}: \mathrm{C}_{6} \mathrm{H}_{4}: \mathrm{NH} \\
& \text { Chinonimid } \\
& \rightarrow\left(\mathrm{NH}_{2} \mathrm{C}_{6} \mathrm{H}_{4}\right)_{2} \mathrm{C}: \mathrm{C}_{6} \mathrm{H}_{4}: \mathrm{NHHCl} \\
& \text { Chinonimoniumsalz, Farbstoffsalz. }
\end{aligned}
$$

Zunächst erfolgt cine Tautomerisation des Carbinols zum Chinol, das als unbeständige Verbindung die Tendenz hat, unter Abspaltung von Wasser in das beständigere Chinonimid überzugehen, das sich mit Säuren zu dem noch beständigeren Chinonimoniumsalz, dem Farbstoff, vereinigt. Diese Auffassung steht auch mit den experimentellen Ergebnissen v. $\mathrm{B}$ a e yers ${ }^{64}$ ) über die Chinonimide in Einklang; im übrigen erinnert sie in vielen Punkten an die von $\mathrm{H}$ a $\mathrm{ntzs}$ ch im AnschluB an die Theorie der $y$ Basen entwickelte.

Auf Grund der vorstehend erwähnten zahlreichen Arbeiten kann es als erwiesen gelten, daB das Auftreten von Farbe bei umlagerungsfähigen Stoffen, z. B. infolge von Salzbildung in vielen Fällen mit der Umlagerung in einem ursächlichen Zusammenhang steht. Die letzte Ursache der Farbigkeit sehen alle bisher genannten Forscher in der durch die Umiagerung geschaffenen neuen Molekularstruktur, die, was z. B. in den Formeln der Chinoide zum Ausdruck gebracht werden soll, einen ungesättigteren Charakter besitzt als die nicht der umgelagerte Verbindung.

VIII. Absorptionsstreifen im Ultraviolett bei Keto-Enoltautomeren. Isorropesis.

Eine andere Ansicht äber das Zustandekommen von Farbe, die schon sus dem Grunde sehr be-

63) Walden, Z. physikal. Chem. 43, 443 ; Gomberg, Berl. Berichte $\mathbf{3} \boldsymbol{\gamma}, 2408$.

64) Berl. Berichte $33^{*}, 2851$. 
achtenswert ist, da sie mehr die physikalische Seite des Problems berücksichtigt, verdankt man $\mathrm{B}$ a l y , dessen Untersuchungen an die eingangs erwähnten Forschungen $\mathrm{Hartloys}$ über Absorptionen im Ultraviolett anknüpfen und der diese besonders gemeinschaftlich mit Desch ${ }^{65}$ ) wesentlich gefördert hat.

Wie früher dargelegt wurde, zeigen aliphatische Verbindungen meist allgemeine Absorption im Ultraviolett, indem alle Strahlen oberhalb einer bestimmten Grenze absorbiert werden. Einige tautomer reagierende Verbindungen machen jedoch hierin eine sehr bemerkenswerte Ausnahme. So geben Acetylaceton: $\mathrm{CH}_{3} \mathrm{COCH}_{2} \mathrm{COCH}_{3}$ und einige Metallverbindungen desselben wie das Be-, Al-, Thi-, Salz Absorptionsstreifen, Acetessigester $\mathrm{CH}_{3} \mathrm{COCH}_{2}$ $\mathrm{COOC}_{2} \mathrm{H}_{5}$ gibt nur allgemeine Absorption ohne Andeutung eines Bandes, während sein Aluminiumderivat ein mit dem des Acetylacetons fast identisches Bandenspectrum liefert. Daß nicht einerseits die Enolgruppierung

$$
\mathrm{CH}=\stackrel{\mathrm{C}-}{ } \underset{\mathrm{OH}}{ }
$$

die im Acetylaceton und seinen Metallderivaten sowie den Metallderivaten des Acetessigesters anzunehmen ist, noch andererseits die Ketogruppierung: $-\mathrm{CH}_{2}-\mathrm{CO}-$ die Entstehung des Absorptionsstreifens veranlaßt, wurde dadurch bewiesen, daß weder der Kohlenstoffester noch der Sauerstoffester des Acetesigesters:

$$
\begin{gathered}
\mathrm{CH}_{3} \mathrm{COCHCO}_{2} \mathrm{C}_{2} \mathrm{H}_{5} \\
\mathrm{CH}_{3}
\end{gathered} \text { und } \begin{gathered}
\mathrm{CH}_{3} \mathrm{C}=\mathrm{CHCOOC}_{2} \mathrm{H}_{5} \\
\mathrm{OCH}_{3}
\end{gathered}
$$

die Erscheinung zeigen; erstere Verbindung ist praktisch vollkommen durchlässig, während letztere nur kontinuierliche Absorption bewirkt. Die Schlußfolgerung, da $\beta$ die freien Wasserstoffverbindungen (Keto- und Enolform) sich gleich verhalten würden, ist deshalb zulässig, weil nach $\mathrm{H}$ a r t 1 e y der Ersatz des Wasserstoffatoms durch niedrige Alkylreste den Typus des Absorptionsspectrums nicht ändert.

Es ist daher sehr wahrscheinlich, daßdie selektive Absorption nicht durch einen bestimmten durch eine Formel darstellbaren Zustand des Molekiuls verursacht wird, sondern der Existenz eines dynamischen Gleichgewichts zwischen zwei in der Lösung vorbandenen Formen der Verbindung zuzuschreiben ist.

Gestützt wird diese Auffassung durch die Cntersuchung des Einflusses von Säuren und Basen auf den Charakter der Absorptionsstrei'en. Nach anderen Untersuchungen ist es wahrscheinlich, daß die Geschwindigkeit der Umwandlung von Keto- in Enolisomere durch Basen erhöht und durch Säuren verzögcrt wird. Konform damit ist der spektroskopische Befund, daß Basen eine Erhöhung der Beständigkeit der Absorptionsstreifen bewirken, $d . h$. die Streifen sind bei Gegenwart von Natron noch bei weit kleineren Schichtdicken sichtbar als ohne Basenzusatz, was besonder's dureh die ron $\mathrm{H}$ a r $\mathbf{r}^{\mathrm{t}}$ le y herrührende graphische Darstellung der Versuchsergebnisse plausibel wird (s. beistehende Fig. 2). In derselben sind auf den Abscissen die den Grenzen

65) Trans. Chem. Soc. 85, 1029; 87, 766; Z. physikal. Chem. 55, $48 \overline{5}$. der Absorption (im Ultraviolett) entsprechenden Schwingungszahlen aufgetragen (reciproke Werte der Wellenlängen) auf den Ordinaten die Logarithmen der Schichtdicken ( $\mathrm{in} \mathrm{mm}$ ) boi der betreffenden Konzentration (in der Regel wurde 0,1 Millimol der Substanz in $50 \mathrm{ccm}$ Alkohol oder Wasser gelöst). So zeigt I die kontinuierliche Alsorption des Acetessigesters, 2 und 3 die unter einander sehr ähnlichen Spektren des Acetylacetons und seines Berylliumderivates. Die Kurven 4 und 5 geben die Lagen der Absorptionsstreifen des Acetessigesters bei Gegenwart von 1 Mol. Alkali sowie bei Uberschuß von Alkali und zeigen, daß lotzterer nicht die Lage des typischen Absorptionsstreifens verändert, wohl aber seine Beständigkeit erhöht.

Ähnliche Verhältnisse wurden beim Acetbernsteinsäureester, Acetondicarbonsäureester, Benzoylaceton, Benzoylessigster und Benzoylbernsteinsäureester beobachtet. Letztere drei zeigen auch im. freinen Zustande Absorptionsstreifon, durch Zusatz von Natron treten diese aber in allen Fällen her vor. Die Resultate sprechen dafür, daß der reversiblen Umwandlung von Keto- Enoltautomeren, die strukturchemisch durch einen Bindungswechsel gedeutet wird:

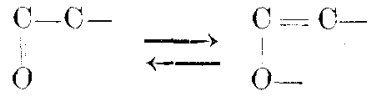

ein bestimm er periodischer Vorgang entspricht, der mit den absorbierten Strahlen synchron ist.

$\mathrm{Zu}$ einer in gewisser Weise befriedigenden Erklärung gelangen Verff. durch Anwendung der E'ektronentheorie auf chemische Systeme, d. h. die Thomsonsche Annahme, dab die chemischen Atome Systeme von Elektronen darstellen. Bei Bildung chemischer Verbindungen entstehen durch Cbertragung eines oder mehrerer Elektronen von einem Atom auf das andere ein oder mehrere Farad a ysche Kraftröhren, deren jede einer Bindungseinheit entspricht. Wird die Anordnung der Kraft-

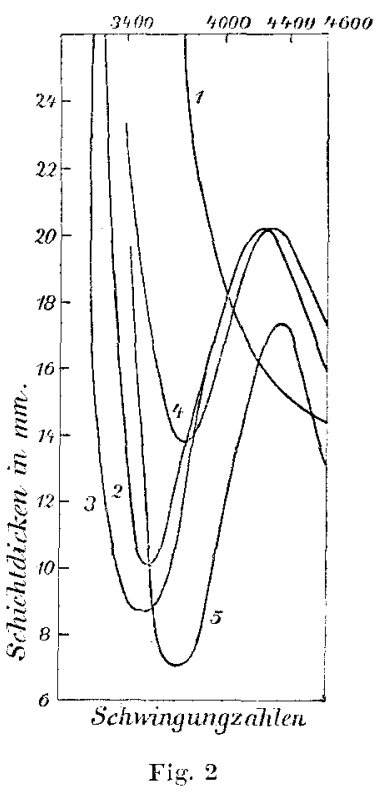

röhren geändert, so treten in dem System Störungen in Form von Schwingungen auf. Die durch obigen Bindungswechsel . verursachten Schwingungen entsprechen denen der Lichtwellen; nach dem Prinzip der Resonnanz wird daher ein System, in welchem solche Schwingungen stattfinden, Lichtstrahlen der betreffenden Wellenlängen absorbicren und da diese Schwingungen nicht synchron sein werden, wird der Absorptionsstreifen meßbare Breite besitzen. 
Spektroskopisch ähnliche Verhältnisse treten auch bei Anwesenheit zweier benachbarter Carbonylgruppen im Molekiil des absorbierenden Stoffes auf. Wie bei den Keto- Enoltautomeren wird auch durch diese Atomgruppierung nach A. W. Stewart und E. C. C. $B a\left(y^{66}\right)$ ein Oscillationszustand bedingt, der aber im Gegensatz zu dem zuerst besprochenen die Absorption von mit größeren. Wellenlängen zur Folge hat (d.h. im Blau), so dab diese Stotfe gelb 'erscheinen. Beim Diacetyl geben die Verff. die Oscillationen dureh folgendes Sehema wieder :

$$
\begin{array}{lll}
\mathrm{CH}_{3}-\mathrm{C}=\mathrm{O} & \longrightarrow \\
\mathrm{CH}_{3}-\mathrm{C}=\mathrm{O} & \longrightarrow \mathrm{CH}_{3}-\mathrm{C}-\mathrm{O} \\
\mathrm{CH}_{3}-\mathrm{C}-\mathrm{O}
\end{array}
$$

und nemmen den die Absorption bedingenden Vorgang Is orropesis das charakteristische $A b$ sorptionsband ein is or r o p is ches.

Ganz analoge Erscheinungen sind bei Chinonen beobachtet. Benzochinon hat ein isorropisches Band und zeigt keine Anzeichen von benzolischer Struktur. Die Rinführung von Methylgruppen sowie von Chlor vermindert die Beständigkeit des isorropischen Bandes und ruft ein benzolisches Band im Spectrum hervor; auch die chemische Reaktionsfähigkeit der Carbonylgruppen scheint mit dem isorropischen Vorgang in Beziehung zu stehen.

Von diesen neuen Methoden der spektroskopischen Konstitutionsbestimmungen sind sicher sowohl für Keto-Enoltautomere als für Chinoide wichtige Einblicke in feinere Isomerieerscheinungen zu erwarten und man muß deshalb den weiteren Arbeiten Balys mit Spannung entgegensehen.

\section{Über die Analysen einiger römischer Gläser und Bronzen.}

\section{Von F. Henrich und P. Roters.}

\section{(Eingeg. d. 21.6. 1907.)}

Vor einiger Zeit erhielt der eine von uns von Herrn Baurat H. J a c o b j jun. in Homburg v. d.H. ein Stückchen intensiv gefärbtes Glas, das bei den Ausgrabungen auf dem Römerkastell Saalburg bei Homburg zutage getreten und sicher römischen Ursprungs war. Eine qualitative Analyse ergab, daß ein Natronglas vorlag, das als Nebenbestandteile Eisen und Mangan enthielt. Um über die Beziehungen beider zur Farbe des Glases etwas zu erfahren, unterwarfen wir eine Anzahl von Glasscherben sicher römischen Ursprungs einer genauen quantitativen Analyse.

Die weitaus meisten römischen Gläser, die man in Nuseen und Sammlungen sieht, sind mehr oder weniger grün gefärbt, und viele zeigen auf ihrer Oberfläche perlmutterartigen Glanz. Die Ansicht, daB den Römern die Herstellung farbloser Gläser unbekannt gewesen sei, ist längst durch Funde bei Ausgrabungen widerlegt. Das antike Glas solite als Rival der Edelsteine bunt sein, so verlangte es lange Zeit die Wode, und erst seit Ende des ersten Jahrhunderts nach Chr. werden die farblosen Gläser

65) Proc. Chem. Soc. 22, 34, 35.

populärer. Bei den Ausgrabungen auf der Saalburg wurde denn auch eine große Anzahl Glasscherben römischen Ursprungs zutage gebracht, die alle Ubergänge von intensiv dunkelgrün gefärbten zu fast farblosen Gläsern darstellen. Von einer Anzah] solcher Scherben unzweifelhaft römischen Ursprungs, die Herr $J$ a c o b i jr. uns gütigst zur Verfügnug stellte, wählten wir vierStücke von ungefähr gleicher Dicke aus. Das cine (I) hatte eine tiof dunkelgrüne, satte Farbe, während ein anderes (IV) in der Durchsicht völlig farblos erschien und nur auf den Bruch. flächen einen schwach grünen Schimmer erkennen lie 3. Von den beiden anderen war das eine (III) in der Durchsicht schwach grün gefärbt, das andere (II) zeigte ein stärkeres Grün, aber nicht von der Intensität wie das zuerst erwähnte. In diesen vier Proben waren die wichtigsten Phasen eines allmählichen Ubergangs von farblos bis zum intensivsten noch durchsichtigen Grün vertreten.

Die Analysen ergaben folgende Resultate:

$$
\begin{aligned}
\text { tief dunkelgrünes Glas (I) } \\
\mathrm{SiO}_{2}=66,54 \% \\
\mathrm{Al}_{2} \mathrm{O}_{3}=1,95 \% \\
\mathrm{Fe}_{2} \mathrm{O}_{3}=1,74 \% \\
\mathrm{CaO}=6,07 \\
\mathrm{MgO}=038 \% \\
\mathrm{Na}_{2} \mathrm{O}=23,73 \\
100,41 \%
\end{aligned}
$$

schwachgr ünes Glas (III)

$$
\begin{aligned}
& \mathrm{SiO}_{2}=68,54 \% \\
& \mathrm{Al}_{2} \mathrm{O}_{3}=1,85 \% \\
& \mathrm{Fe}_{2} \mathrm{O}_{3}=0,48 \% \\
& \mathrm{CaO}=6,45 \% \\
& \mathrm{MgO}=0,65 \% \\
& \mathrm{Na}_{2} \mathrm{O}=21,61 \\
& -\frac{99,58}{2}
\end{aligned}
$$$$
\text { grünes Glas (II) }
$$$$
\mathrm{SiO}_{2}=69,44 \%
$$$$
\mathrm{AI}_{2} \mathrm{O}_{3}=3,79 \%
$$$$
\mathrm{Fc}_{2} \mathrm{O}_{3}=0,82 \%
$$$$
\mathrm{CaO}=6.53 \%
$$$$
\mathrm{MgO}=0,63 \%
$$$$
\mathrm{Na}_{2} \mathrm{O}=\frac{18,71 \%}{99,92}
$$

farbloses Glas (TV) $\mathrm{SiO}_{2}=67,00 \%$ $\mathrm{Al}_{2} \mathrm{O}_{3}=4,21 \%$ $\mathrm{Fe}_{2} \mathrm{O}_{3}=0,49 \%$ $\mathrm{CaO}=6,75 \%$ $\mathrm{MgO}=0,54 \%$ $\mathrm{MnO}=0,36 \%$ $\mathrm{Na}_{2} \mathrm{O}=20,67 \%$ 100,02

Man sieht, daß überall Natrongläser vorlagen, und daß von den Ingredienzien, die die Farbe bedingen, der Eisengehalt bei den ersten drei Gläsern mit der Intensität der grünen Färbung abnimmt.

Um dies Resultat zu kontrollieren, suchte ich an den zur Verfügung stehonden Scherben zwei weitere Stichproben aus, ein schwach und ein etwas stärker hellgrün gefärbtes Glas. Die Analysen ergaben folgendes Resultat:

schwach hellgrïn
gefürbtes Glas $(\mathrm{Y})$
$\mathrm{SiO}_{2}=67,78 \%$
$\mathrm{Al}_{2} \mathrm{O}_{3}=2,12 \%$
$\mathrm{Fe}_{2} \mathrm{O}_{3}=0,48 \%$
$\mathrm{CaO}=6,74 \%$
$\begin{aligned} \mathrm{MgO} & =0,64 \% \\ \mathrm{Na} & =21,72 \% \\ & =99,48\end{aligned}$

$$
\begin{gathered}
\text { etwas stärker hellgrim } \\
\text { gefärtotes } \mathrm{Glas}(\mathrm{IV}) \\
\mathrm{SiO}_{2}=67,55 \% \\
\mathrm{Al}_{2} \mathrm{O}_{3}=4,08 \% \\
\mathrm{Fe}_{2} \mathrm{O}_{3}=0,59 \% \\
\mathrm{CaO}=6,74 \% \\
\mathrm{MgO}=0,54 \% \\
\mathrm{Na} \mathrm{O}=20,67 \% \\
=100,17 \%
\end{gathered}
$$

also eine Bestätigung des oben Gefundenen.

Damit ist auch experimentell bewiesen, was von vornherein zu erwarten war, daß nämlich die grüne Farbe der römischen Gläser in der Hauptsache durch kieselsaures Eisenoxydul bedingt wird.

Aber noch in einer andern Hinsicht sind die Analysen lehrreich. In allen Glasscherben konnte man neben Eisen auch Mangan nachweisen. Als aber von jeder Probe je ein Gramm der quantitativen Analyse unterworfen wurde, war da das Mangan in 